\title{
Multi-fault feature extraction and diagnosis of gear transmission system using time-frequency analysis and wavelet threshold de-noising based on EMD
}

\author{
Renping Shao*, Wentao Hu and Jing Li \\ School of Mechatronics, Northwestern Polytechnical University, Xi'an, Shaanxi, China
}

Received 26 January 2012

Revised 4 August 2012

Accepted 19 October 2012

\begin{abstract}
A gear transmission system is a complex non-stationary and nonlinear time-varying coupling system. When faults occur on gear system, it is difficult to extract the fault feature. This paper researches the threshold principle in the process of using the wavelet transform to de-noise the system, and combines EMD (empirical mode decomposition) with wavelet threshold de-noising to solve the problem. The wavelet threshold de-noising is acts on the high-frequency IMF (Intrinsic Mode Function) component of the signal, and does overcome the defect by simply highlighting the fault feature. On this basis, the pre-processed signal is analyzed by the method of time-frequency analysis to extract the feature of the signal. The result shows that the SNR (signal-noise ratio) of the signal was largely improved, and the fault feature of the signal can therefore be effectively extracted. Combined with time-frequency analyses in the different running conditions (300 rpm, $900 \mathrm{rpm}$ ), various faults such as tooth root crack, tooth wear and multi-fault can be identified effectively. Based on this theory and combining the merits of MATLAB and $\mathrm{VC}++$, a multi-functional gear fault diagnosis software system is successfully exploited.
\end{abstract}

Keywords: Gear system, wavelet threshold de-noising, empirical mode decomposition (EMD), time-frequency analysis, fault diagnosis, virtual instrument (VI)

\section{Introduction}

As a complex flexibility system, a gear transmission system will vibrate in internal and external incentives. Due to the influence of the nonlinear time-varying characteristics of gear mesh stiffness and the nonlinear stiffness of a supporting system, such as a bearing or gearbox, a complex coupling nonlinear vibration will occur in the system [1, 2 ]. When the fault occurs, the coupling nonlinear vibration characteristics will be more intense, and according to the different locations and types of the faults, the different degree second-order phase and high-order phase coupling phenomenon will be produced [3]. The result of using the common method of signal processing to analyze the system is often not ideal, and the common method of signal processing makes extracting, identifying and diagnosing the fault feature difficult [4].

${ }^{*}$ Corresponding author: Renping Shao, School of Mechatronics, Northwestern Polytechnical University, 127 Youyi St, Xi'an 710072, Shaanxi, China. E-mail: shaorp@nwpu.edu.cn. 
Most signals in the process of fault diagnosis are non-stationary; the signal itself and the related statistics are functions that change following the time. The traditional time domain analysis and the frequency domain analysis do not have the same aspect characteristics of the signal (for example, the relationship between the time and the change of signal spectrum). But the time-frequency analysis can connect with the changes of the signal in the time domain and the frequency domain to process a signal. By this method, it can describe the change rule of the signal's frequency, energy and intensity following time, and can objectively grasp the signal characteristics of the mechanical system fault [5].

Wavelet analysis is a time-frequency analysis method; it can automatically adjust resolution depending on the different frequency components of a signal [6]. For frequency components, the refinable sampling steps are adopted in time domain or frequency domain, so they can focus on any details of the object (function, signal, image, etc) and analyze them. Due to the microscope characteristics of wavelet analysis, it shows the huge superiority in decomposing and reconstructing a signal, separating noise from a signal and extracting the signal feature and other projects. The signal and noise display the different decomposition characteristics in the process of wavelet transform. With the scale of decomposition increasing, the amplitude of the wavelet coefficient corresponding to the signal and containing the important information of the signal is larger but the number is smaller, and while the wavelet coefficient corresponding to noise appears as uniform distribution, the number is larger and the amplitude is smaller. Based on these characteristics of the wavelet transform, the signal-mixed noise can be de-noised. Choosing an appropriate threshold value to process wavelet coefficient, the wavelet coefficient of the signal can be kept and the wavelet coefficient of most noise will be reduced to zero.

At present, the method of wavelet analysis has been used in signal processing, dynamic characteristics identification and damage detection and diagnosis [7]. In regards to the wavelet analysis, the noise produced by tools can be used to detect and diagnose the machine tool state [8]. The wavelet analysis sets a foundation for using the technology of ultrasonic wave to detect the health condition of steel strand [9]. While working on the wavelet transform, Yang Tielin investigated the pressure signal of a pump outlet to discern the fault diagnosis of the pump [10]. But the threshold choice of the wavelet de-noising is difficult. When the SNR is large, a fixed threshold is chosen. When the SNR is small or the signal has a large disturbance, the threshold is determined by Stein's unbiased estimation theory. However, due to the characteristics of the wavelet threshold, this principle will still eliminate some real signals.

EMD is a new time-frequency analysis and statistic transform method which can decompose the signal into a series of decomposition signals from high frequency to low frequency. Each level signal retains the characteristics of data itself. By this method, it not only extracts the signal feature easily but it also highlights the characteristic of damage and fault. At present, the EMD method has been widely applied to signal processing, dynamic characteristics identification, damage detection and fault diagnosis [11], such as the fault diagnosis based on EMD of rotator bearings [12], and the harmonic fault diagnosis in large rotating machinery [13,14]. However, there is a lack in study on the damage detection of gear systems, so there is a huge research space at present. Sometimes the EMD method may regard the weak feature signal as noise, and eliminate it. For example, the endpoint effect. So there are some defects in dealing with the weak feature signal and the complex random, non-stationary signal.

Therefore, in this paper, EMD and the wavelet threshold de-noising are combined friendly for de-noising preprocess. Engineering experience shows that the noise generally locates in the high-frequency part of the signal. The IMF components distribute from high frequency to low frequency, using the wavelet threshold to reduce the noise of the high-frequency components. It then uses de-noised data and the low-frequency components without noise to reconstruct the original signal. By this method, the wavelet threshold de-noising is not acted on the whole signal but only the high-frequency component. This method largely overcomes the defect of using wavelet threshold de-noising [15].

The short-time Fourier transform [16] is an excellent time-frequency analysis method; a non-stationary process is considered as a series of short-time stationary signals, so any short-time signal can be analyzed by the Fourier transform. Gabor introduced the concept of window Fourier transform by using a time-window that can slip on a time axis to achieve a better local analysis of signal in time domain and frequency domain. Recently, many scholars have investigated research on short-time Fourier transform. For instance, Zhou Dan in Chong Qing university, China, proposed research into the short-time Fourier transform being used to analyze the pulse signal [17]; Kuldip used the short-time Fourier transform to identify the language testing signal [18]; and Debbal used the short-time Fourier transform to analyze the heartbeat signal [19]. The signal of a gear transmission system is a time-varying and nonstationary signal; when a fault occurs, the characteristic of the signal will be complex, so it is more appropriate 
to give a non-stationary and time-varying analysis to the fault signals. In this paper, we give the de-noised signal a time-frequency analysis by the short-time Fourier method, which also extracts information of different faults in different speeds, such as tooth root crack, pitch circle crack, and tooth wear, and can effectively identify complex fault.

This paper proposes a gear feature extraction and fault diagnosis method, which combines the time-frequency analysis with the wavelet threshold de-noising based on EMD. In the gear system testing, the various vibration signals produced by faults such as tooth root crack, pitch circle crack, tooth wear and multi-fault are picked up, and the de-noising, feature extraction and fault diagnosis are studied by the new method. Signals will be decomposed into six levels by EMD (combining the high frequency component de-noised by wavelet threshold with the low frequency component without noise) to successfully reconstruct the original signal, and then give the reconstructed signal a time frequency analysis to identify the different faults of the gear system under different running conditions. Thereby, it provides a reliable and effective method for dynamic property identification and damage detection of a complex mechanical system.

Virtual Instrument (VI) is a combination of modern computerization and instrumentation [20]. It has functions of a computer, such as computing, storage, playback, call, display, document management, and complex information processing, and it achieves the specialized function of a traditional instrument by software. The new instrument whose appearance and function are completely identical with the traditional hardware instrument fully utilizes the computer intelligence resource. At present, many virtual instruments have been developed to measure, display, diagnose and control in actual engineering (for example, using virtual instruments to study the measurement of aircraft inverter output characteristics [21]); the requirements of online upgrade and adjustment function are proposed based on the virtual instrument assembly language of XML [22]. But the virtual instrument for the detection and diagnosis of a gear transmission system is less. Therefore, this paper will develop the algorithm of fault diagnosis and feature extraction which combines the wavelet threshold de-noising based on EMD with the time frequency analysis. The numerical computing, signal processing, graphics display, fault feature extraction and diagnosis are accomplished in MATLAB. Using COM (Component Object Module) technology, it changes the program of each signal analysis and processing algorithm written in MATLAB into COM module for $\mathrm{VC}++$ calls to improve the efficiency of system development. Using $\mathrm{VC}++$ to design the main interface and achieve sub-interface calls, the software system of gear fault analysis and diagnosis is developed.

\section{The theory of de-noise preprocessing}

\subsection{Wavelet threshold de-noising}

In practical engineering, the useful signal usually appears as a low-frequency component and a stationary signal, while the noise signal appears as a high frequency signal. So a de-noising method is propounded as follows: decompose the signal and process the wavelet coefficient through the form of threshold, then reconstruct the signal to achieve the purpose of de-noising. The de-noising process of a one-dimensional signal is divided into three steps: (1) choose a wavelet and determine the decomposition level $N$, then decompose the signal into $N$-level; (2) from first level to $N$-level, for the high-frequency coefficient of each level, choose a threshold to give them a threshold quantitative process; (3) according to the $N$-level low-frequency coefficients from the wavelet decomposition and high-frequency coefficients given quantitative processes from the first level to the $N$-level, reconstruct the one-dimensional signal.

The general rule of selecting threshold is as follows [23]:

(1) Fixed threshold. The threshold is $\lambda_{1}=\sigma_{n} \sqrt{2 \ln N}$, where $\sigma_{n}$ is the standard variance of noise, and $N$ is the signal length. By this method, the threshold will always be too large. As a result, some useful signals will be eliminated.

(2) Self-adaptive threshold, based on the theory of Stein's unbiased likelihood estimation. The threshold is $\lambda_{2}=$ $\sigma_{n} \sqrt{w_{b}}$, where $\sigma_{n}$ is the standard variance of noise, and $w_{b}$ is the venture function. In this method the threshold is relatively small, so the useful signal is not easy to eliminate. 
(3) Heuristic threshold. It is not only the integration of the first two methods but also the best threshold selection. To be specific, the first method is used when the SNR is large. When the SNR is small or has a large disturbance, the second method is used. In this paper, this threshold is used. Assuming $s$ is the sum of the square of the $n$ wavelet coefficients, make $\eta=(s-n) / n, \mu=\left(\log _{2} n\right)^{3 / 2} \sqrt{n}$.

$$
\lambda_{3}= \begin{cases}\lambda_{1} & \eta<\mu \\ \min \left(\lambda_{1}, \lambda_{2}\right) & \eta>\mu\end{cases}
$$

In practical application, due to the characteristics of wavelet threshold de-noising, this rule will still eliminate some real signals.

The method of wavelet threshold de-noising used in this paper is as follows:

$$
\hat{y}=T_{s}(X, \lambda)= \begin{cases}\operatorname{sgn}(X)(|X|-\lambda) & |X| \geqslant \lambda \\ 0 & |X|<\lambda\end{cases}
$$

Where $X$ is the wavelet coefficient before processing, $\hat{y}$ is the processed wavelet coefficient. $\lambda$ is a threshold; $T_{s}$ is a threshold function. It can be seen from the Eq. (2) that if the amplitude is less than $\lambda$, the wavelet coefficients will be zero. Contrarily, the wavelet coefficients will be completely retained, or make a corresponding "shrink". Finally, the inverse wavelet transform is processed by utilizing wavelet coefficients processed to reconstruct the new useful signal.

\subsection{The wavelet threshold de-noising based on EMD}

An empirical mode decomposition method has three assumptions [24,25]: (1) signal has at least one maximum point and one minimum point; (2) the scale of characteristic time is defined by the time-lapse of extremum points; (3) If the whole signal only contains the twist point and but not the extremum point, the extremum points can be found by taking differential to the signal one time or more times, and then the final result will be obtained by integrating all the components. Thus any signal can be decomposed into the sum of many IMFs (intrinsic mode function), and each IMF can satisfy the following conditions: (a) in the signal, the number of zero points is equal to the number of extremum points or the difference is less than one; (b) at any point of signal, the mean value of envelope determined by the local maximum points and the local minimum points is zero, namely, the signal is local symmetry on the time-axis.

The detailed steps of the empirical mode decomposition:

(1) Determine all maximum and minimum points of $s(t)$, and then all points are connected by two smooth curves which are respectively called the upper envelope and the lower envelope; all the signals are included between them, the upper envelope and the lower envelope. Calculated the average value of the two curves, denoted as $m_{1}(t)$, with $s(t)$ minus $m_{1}(t)$ to obtain:

$$
s(t)-m_{1}(t)=h_{1}(t)
$$

If $h_{1}(t)$ is an intrinsic mode component, then $h_{1}(t)$ is the first component of $s(t)$.

(2) Usually, $h_{1}(t)$ does not satisfy the conditions of intrinsic mode function, so consider $h_{1}(t)$ as an original signal. Repeat the step (1), repeat screening $k$ times, and obtain $h_{1 k}(t)=h_{1(k-1)}(t)-m_{1 k}(t)$ until the $h_{1 k}(t)$ becomes an IMF, which is denoted as $c_{1}(t)=h_{1 k}(t)$. So $c_{1}(t)$ is the first intrinsic mode function satisfying the conditions.

(3) $c_{1}(t)$ should contain the shortest or the most elaborate component of the signal, separate $c_{1}(t)$ from $s(t)$ to obtain:

$$
r_{1}(t)=s(t)-c_{1}(t)
$$


Considering $r_{1}(t)$ as the original data, and repeat steps (1) and (2). Then the second intrinsic mode function of $s(t)$ satisfying the conditions will be obtained, which is denoted as $c_{2}(t)$. The $n$ intrinsic mode functions of $s(t)$ will be obtained after repeating $N$ times, namely

$$
\left\{\begin{array}{l}
r_{1}(t)-c_{2}(t)=r_{2}(t) \\
\ldots \ldots \ldots \\
r_{n-1}(t)-c_{n}(t)=r_{n}
\end{array}\right.
$$

The whole process does not stop until it encounters any one of the following guidelines: (1) the component $c_{n}(t)$ or the residual $r_{n}(t)$ is small enough; or (2) the residual $r_{n}(t)$ is a monotone function from which intrinsic mode components satisfying the conditions can't be extracted again. From Eqs (4) and (5), obtain.

$$
s(t)=\sum_{j=1}^{n} c_{j}(t)+r_{n}(t)
$$

Therefore, any one signal $s(t)$ can be decomposed into $N$ intrinsic mode functions and one residual $r_{n}(t)$, from high to low. Different frequency components of signal are contained in $c_{1}(t), c_{2}(t), \ldots c_{n}(t)$, which will alter with the change of the signal itself. Usually, the most distinct and important information of the original signal is focused on the first few IMFs obtained by the EMD method.

Due to the defect of simply using the method of wavelet threshold de-noising, EMD and wavelet threshold denoising are friendly combination for de-noising pre-processing. Engineering experience shows that the noise generally locates in the high-frequency part; the IMF components just distribute from high frequency to low frequency. If directly giving up the high-frequency IMF components and then reconstructing the original signal to achieve denoising, the useful information existing in high frequency IMF components will be abandoned. So our idea is to use the wavelet threshold de-noising to process the high-frequency IMF components with noise, and combine the high-frequency IMF components obtained with the low-frequency IMF components without noise to reconstruct the original signal. As a result, the wavelet threshold de-noising does not act on the whole signal but only the highfrequency IMF components, which largely overcomes the shortcoming of simply using wavelet threshold de-noising and effectively retains the characteristics of the original signal.

\section{Theory of time-frequency analysis}

The time-frequency analysis is a very effective method for analyzing the non-stationary signal, and the short-time Fourier transform (STFT) is one of the most commonly used time-frequency analysis [26]. For the non-stationary signal $s(t)$, assuming $h(t)$ is a window function with short time-width which slides along the time axis, the shorttime Fourier transform of the signal $s(t)$ is defined as:

$$
S(t, w)=\operatorname{STFT}_{s}(t, w)=\int_{-\infty}^{+\infty} s(\tau) h *(\tau-t) e^{-j w \tau} d \tau
$$

where * represents complex conjugate. In fact, it is the time shift and frequency shift of the window function $h(t)$ that make the Fourier transform have a local feature, which is a function of both time and frequency.

In fact, $S(t, w)$ is the inner product of basis function $h_{t . w}(\tau)$, which is the modulation form of time-shift and frequency-shift of window functions $h(t)$ and the signal $s(t)$.

$$
S(t, w)=\left\langle s(\tau), h(\tau-t) e^{j w \tau}\right\rangle
$$

In principle, the window function $h(t)$ can be freely chosen in a square-integral space. However, in practice, in order to make the integral of Eq. (7) only affected by $s(t)$ and its vicinity value, it's hoped that the window function is a narrow time-function and the frequency spectrum function of $h(t)$ is also a narrow function. According 


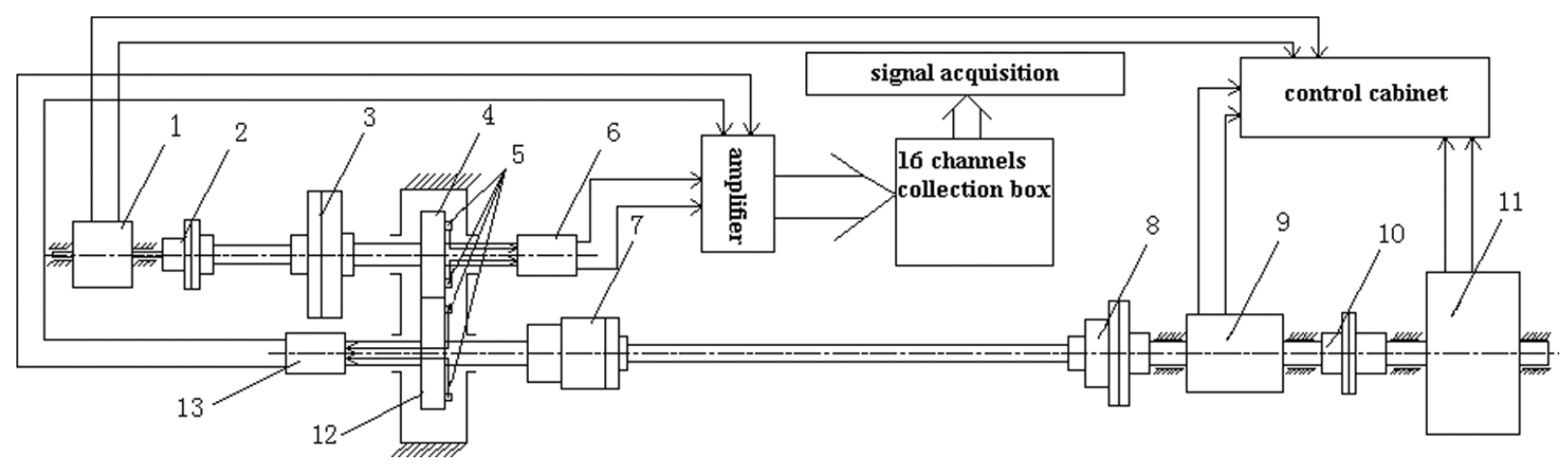

Fig. 1. The testing equipment of a fault gear system, 1. motor; 2. small coupling; 3. big coupling; 4. small gear; 5. acceleration sensor; 6. transmission ring; 7. gear coupling; 8. gear coupling; 9. torque and speed sensor; 10. coupling; 11. magnetic loader; 12. big gear; 13. transmission ring.

to the uncertainty principle, the time width and the bandwidth of the signal may not be arbitrarily small at the same time. The main purpose of applying the STFT is to grasp the local frequency characteristic of the signal, but introducing the window function will reduce the local resolution. For a non-stationary signal, the local spectrum is time varying; the excessively wide window function will confuse the adjacent spectrum and not display the correct local spectrum - that's to say, the width of window function should be fit for the local stable length of signal.

Give Eq. (7) a modulus square processing:

$$
P_{S P}(t, w)=|S(t, w)|^{2}=\left|\int_{-\infty}^{+\infty} s(\tau) h *(\tau-t) e^{-j w \tau} d \tau\right|^{2}
$$

$P_{s p}(t, w)$ is called as short-time Fourier spectrum of $s(t)$; that's the energy spectral density of the original signal at the $t$ moment. Obviously, the spectrum is positive and real constantly.

\section{Feature extraction and diagnosis of gear transmission system}

\subsection{Establishment of the testing equipment and acquisition of the characteristics signal}

A testing diagram of a gear system is shown in Fig. 1. The whole system is driven by motor, through the coupling, the power will been transferred to a reducer gear after the reducer outputting through gear coupling, torque and speed sensor, and the power will be transferred to the magnetic powder loader. Install the acceleration sensor, which sits in a position symmetry to the fault gear, on the testing gear. In addition, the transmission rings, through which the signal measured by the acceleration sensors that turn together with gears are completely transferred out, are placed on the shaft end. During the testing, the size of the torque and the speed are observed and controlled by the control cabinet, and the load applied on the system is controlled by the magnetic powder loader. The running conditions of the gear system are set as four speed: $300 \mathrm{rpm}, 900 \mathrm{rpm}, 1200 \mathrm{rpm}, 1500 \mathrm{rpm}$. Six kinds of gear fault are set on the driving gear; they are tooth root short crack, tooth root long crack, the pitch circle short crack, the pitch circle long crack, tooth wear, and multi-fault (tooth crack and tooth wear fault). In testing, the CRAS signal acquisition software system is adopted for the signal acquisition and analysis, and the above algorithms are used in feature extraction and fault diagnosis.

\subsection{The de-noising process of testing signal}

According to the above thoughts, in order to overcome the defect of using the method of wavelet threshold denoising simply, EMD and wavelet threshold de-noising are friendly combination for de-noising pre-processing. At first, the system signal collected from the experiment is decomposed into many levels by EMD. According to the 

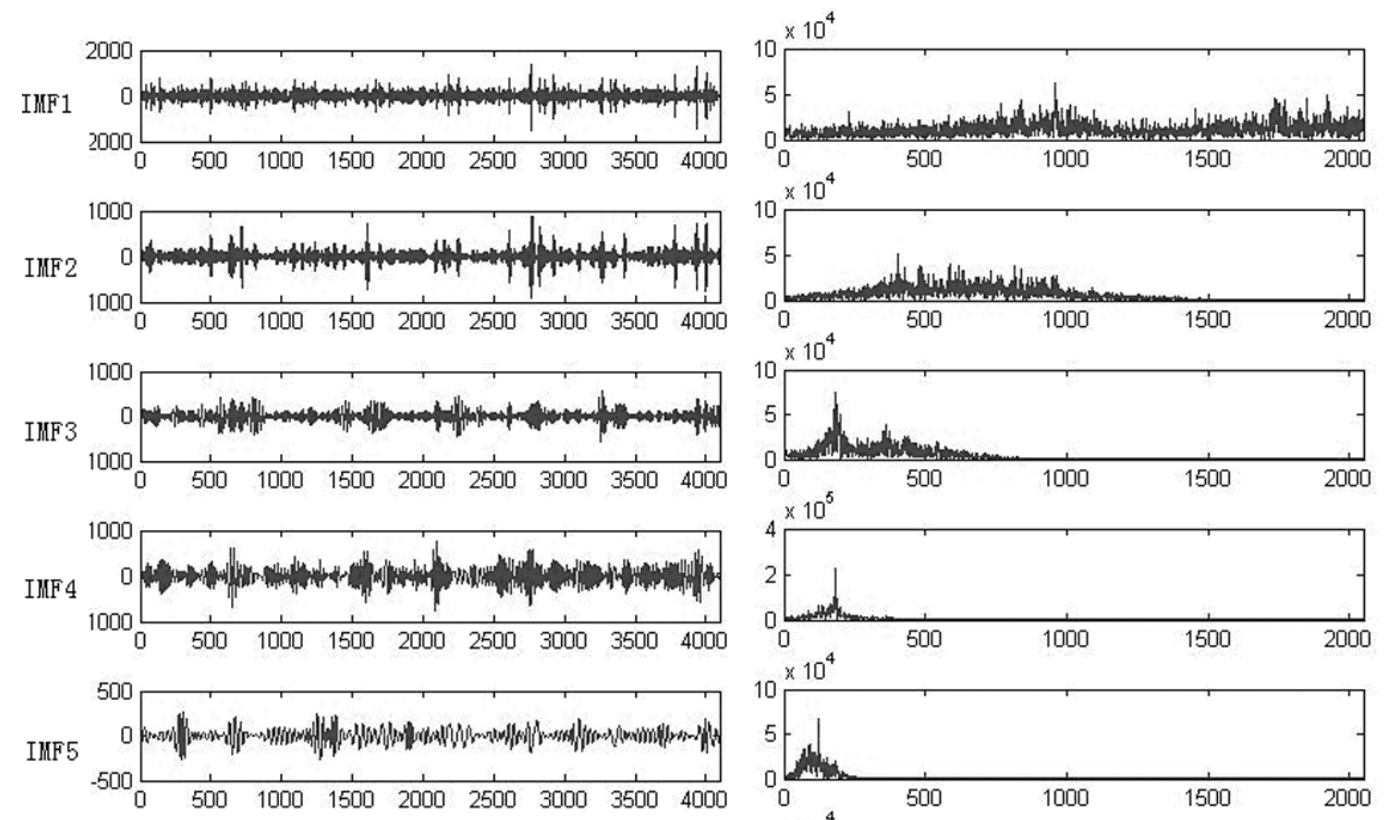

IMF6

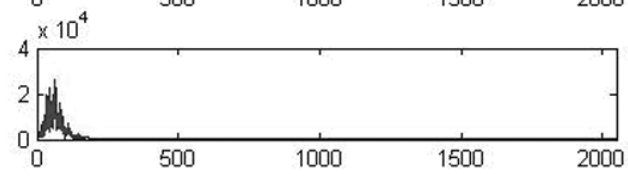

Fig. 2. First six level IMF and spectrum of a crack fault signal at $300 \mathrm{rpm}$.

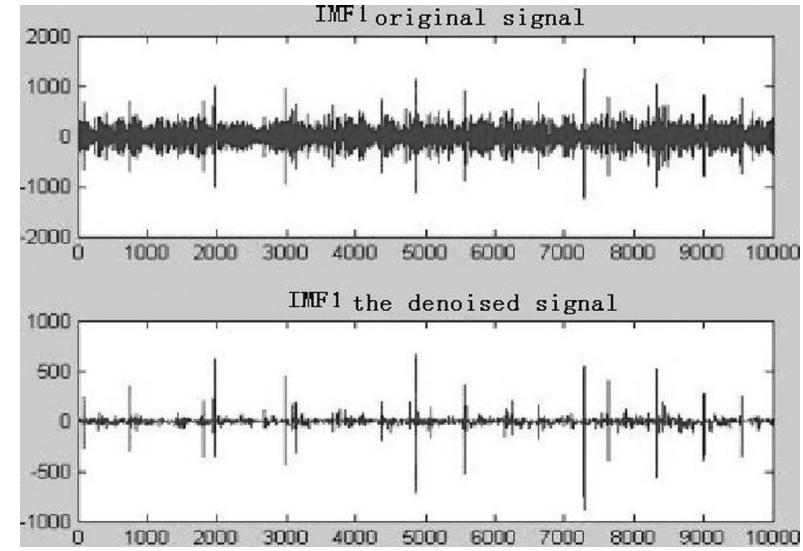

(a) The wavelet threshold de-noising of IMF1

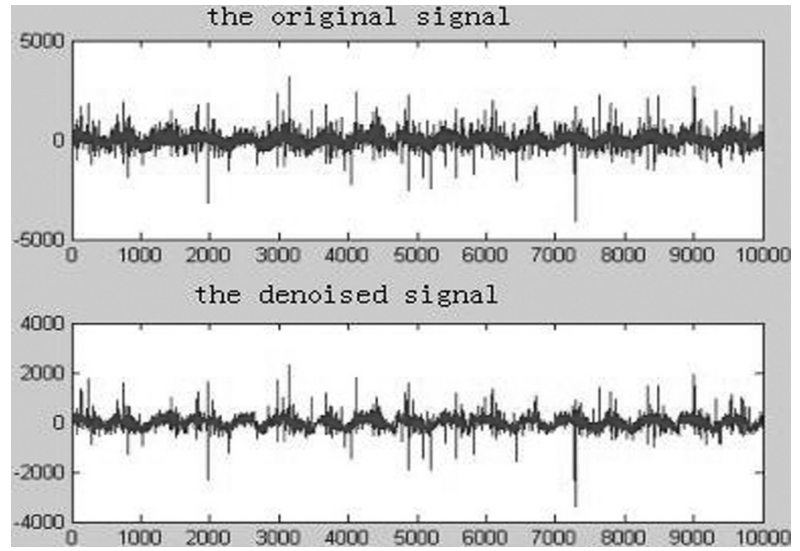

(b) The reconstruction of the de-noised signal

Fig. 3. The wavelet threshold de-noising based on EMD for a crack fault signal at $300 \mathrm{rpm}$.

engineering experience, it is known that the noise is usually the high-frequency signal. The IMF components only distribute from high frequency to low frequency. In the state of $300 \mathrm{rpm}$, the first six level IMF components and their spectrum diagram are shown in Fig. 2. From Fig. 2, it can be seen that the spectral characteristics of high frequency components IMF1 is similar to the noise spectrum. If the high-frequency IMF components are directly given up and the original signal is reconstructed to de-noise, the useful information existing in the high frequency IMF component will lose. The high-frequency IMF components without noise that will be obtained by the wavelet threshold de-noising, combined with the low-frequency IMF component without noise to reconstruct the original signal. The wavelet threshold de-noising does not act directly on the whole signal, but only the high-frequency 

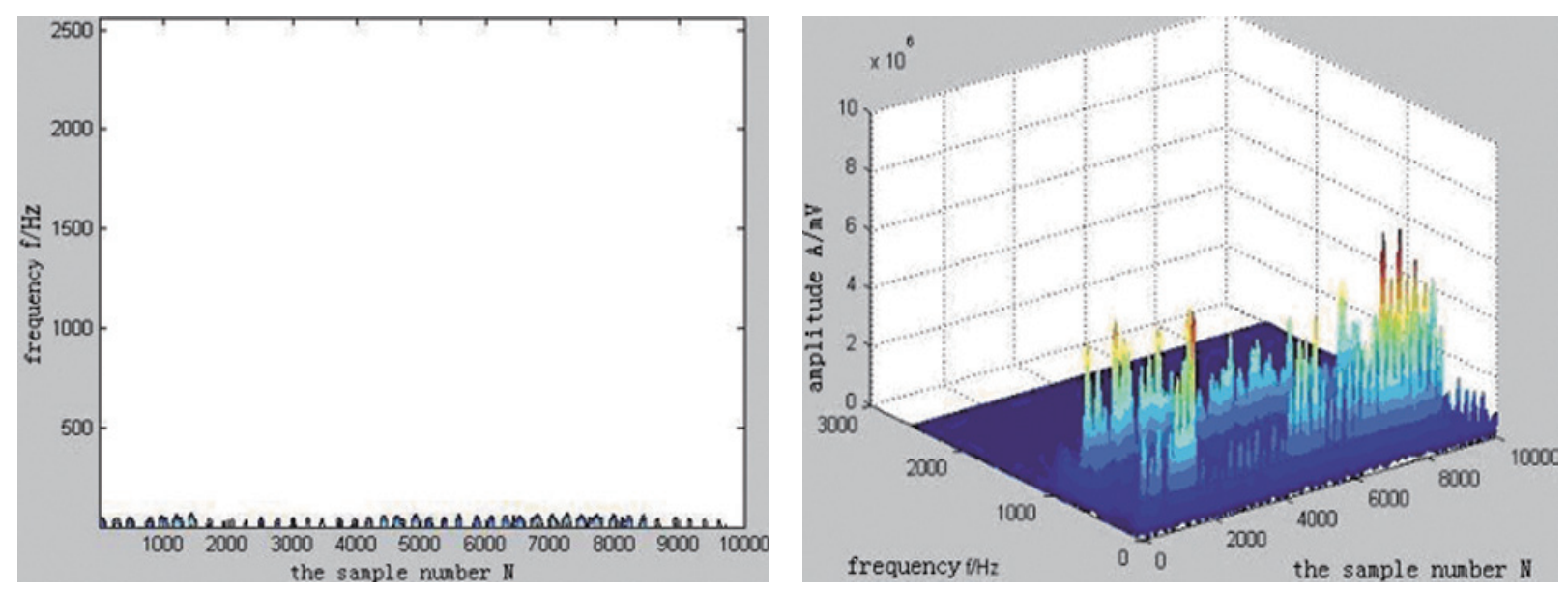

(a) The pre-process result of simply using the wavelet threshold de-noising
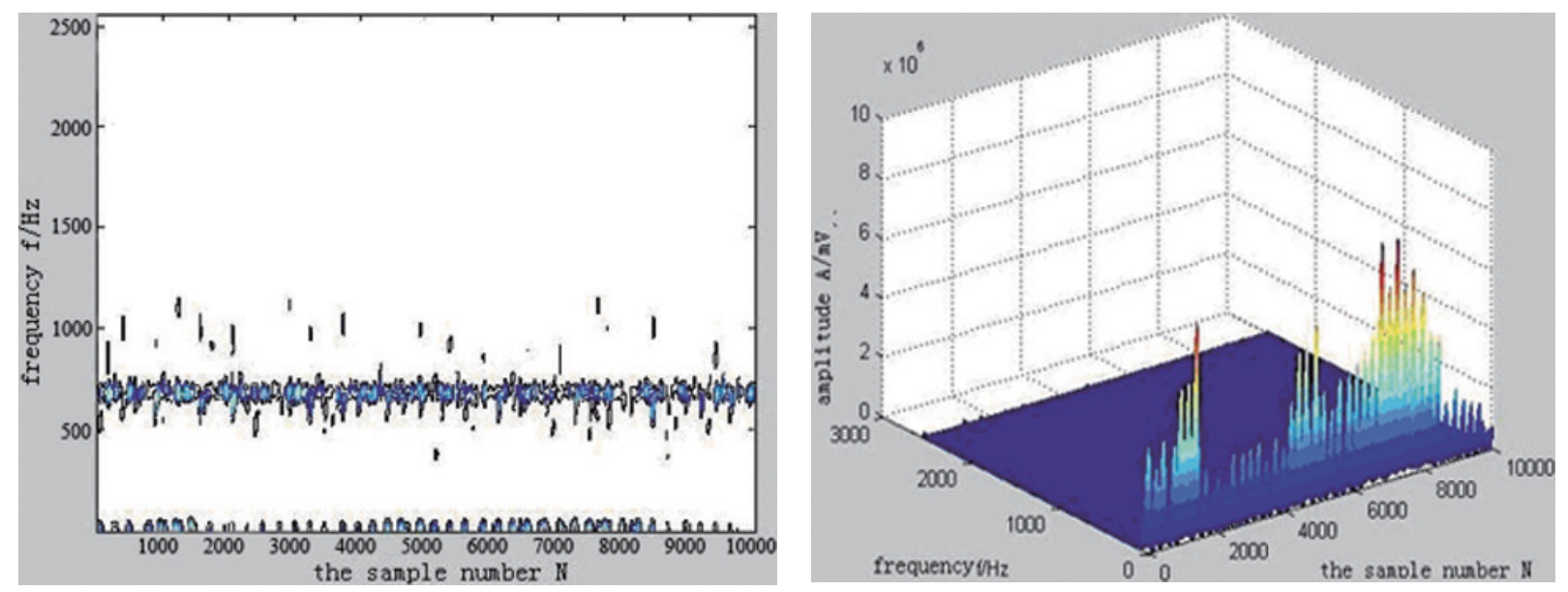

(b) The result of the wavelet threshold de-noising based on EMD

Fig. 4. The result of a time-frequency analysis for a tooth root crack fault signal at $900 \mathrm{rpm}$.

IMF components, which in turn largely overcomes the shortcoming of wavelet threshold de-noising. As shown in Fig. 3, (a) is a de-noising result of the high frequency IMF1 component, which is obtained by decomposing the crack signal in $300 \mathrm{rpm}$. (b) is the reconstruction result of the de-noised signal. As seen from Fig. 3, the denoising effect of the high-frequency IMF1 component by the wavelet threshold de-noising is obvious. Meanwhile, the reconstructed signal shows that it not only retains the original characteristics of the signal but also makes these features more obvious, which is convenient for following analysis. Figure 4 shows the time-frequency analysis result (two-dimensional and three-dimensional distribution) of the tooth root crack signal in the state of $900 \mathrm{rpm}$. It can be seen from Fig. 4(a) that some useful information is eliminated by simply using wavelet threshold de-noising, while this part of useful information is kept by the wavelet threshold de-noising based on the EMD in Fig. 4(b).

Due to the random noise usually mixed in the feature signal, the high- and low-layer frequency bands will appear after giving the signal a multi-layer EMD. The noise always locates in high frequency areas, so the method commonly used in engineering projects is applied to evaluate the SNR of testing data. Namely, to set a threshold (generally it is half of RMS (root-mean-square) of all the data), then analyze these signals, including original signal. The wavelet threshold de-noising signal and the signal processed by wavelet threshold de-noising based on EMD, the part of data which are higher than the threshold or equal to the threshold, are seen as the useful signals; the rest are seen as the noise. Therefore, the SNR will be finally estimated. As shown in Table 1, the table respectively shows the calculated result of SNR about the de-nosing processing of no fault, tooth root crack, pitch circle crack and tooth wear at the state of $300 \mathrm{rpm}$ and $900 \mathrm{rpm}$. 
Table 1

The SNR for various faults in different running conditions

\begin{tabular}{|c|c|c|c|c|c|c|c|c|}
\hline & \multicolumn{4}{|c|}{$300 \mathrm{rpm}$} & \multicolumn{4}{|c|}{$900 \mathrm{rpm}$} \\
\hline & No fault & $\begin{array}{c}\text { Tooth root } \\
\text { crack }\end{array}$ & $\begin{array}{l}\text { Pitch circle } \\
\text { crack }\end{array}$ & $\begin{array}{l}\text { Tooth } \\
\text { wear }\end{array}$ & No fault & $\begin{array}{c}\text { Tooth root } \\
\text { crack }\end{array}$ & $\begin{array}{l}\text { Pitch circle } \\
\text { crack }\end{array}$ & $\begin{array}{l}\text { Tooth } \\
\text { wear }\end{array}$ \\
\hline Original signal & 16.8776 & 22.0128 & 18.1747 & 14.1786 & 12.8019 & 20.3685 & 14.7775 & 10.3097 \\
\hline Wavelet threshold de-noising & 29.3592 & 34.3943 & 23.6825 & 15.4147 & 19.5642 & 40.4246 & 23.7414 & 15.6322 \\
\hline Wavelet threshold de-noising based on EMD & 34.5376 & 37.9479 & 27.4829 & 18.0163 & 19.4409 & 49.4387 & 27.4578 & 16.0879 \\
\hline
\end{tabular}

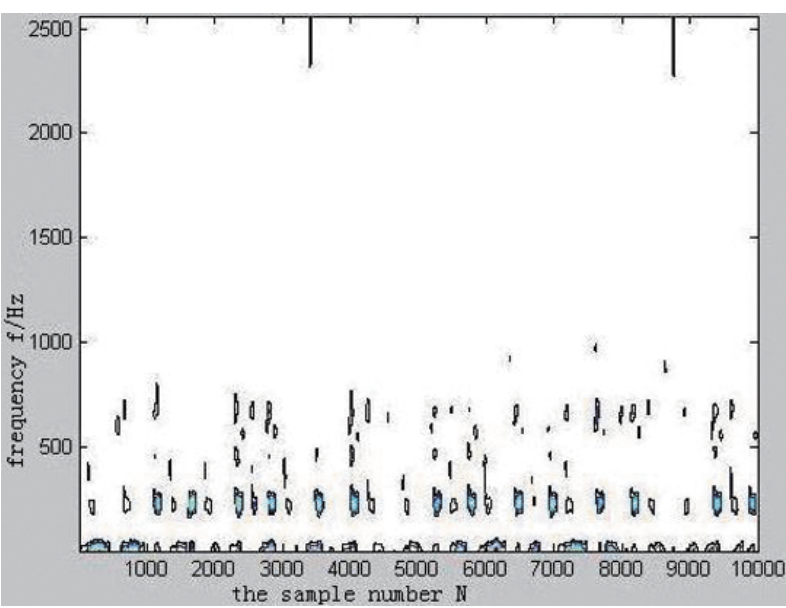

(a) Two-dimensional spectrum of the time-frequency analysis

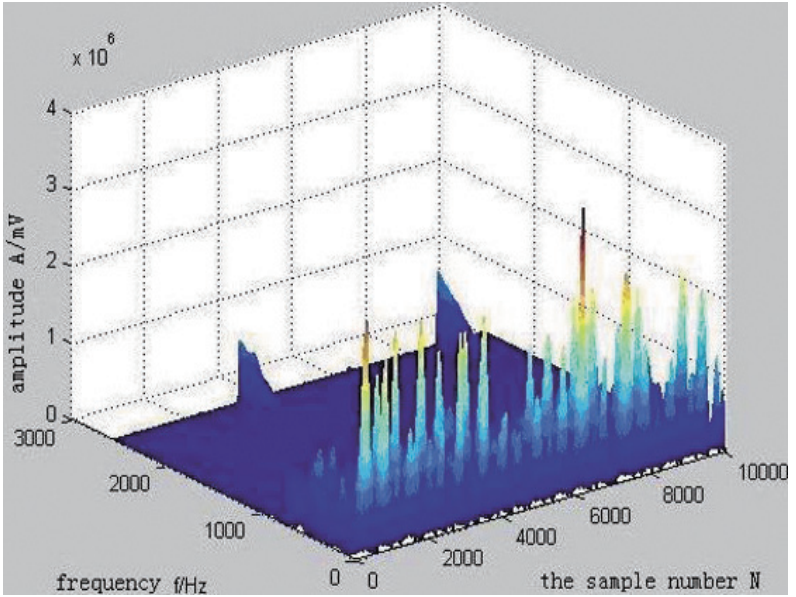

(b) Three-dimensional spectrum of the time-frequency analysis

Fig. 5. The time frequency analysis of a no fault signal at $300 \mathrm{rpm}$.

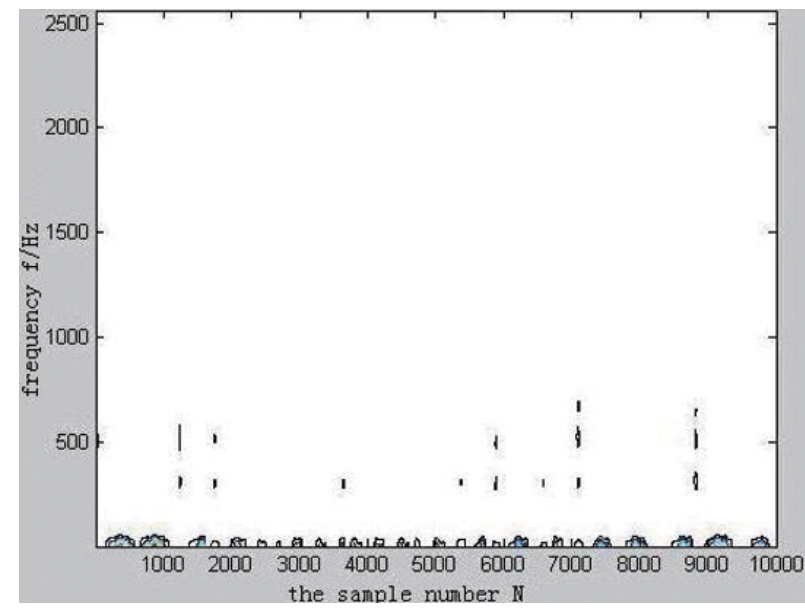

(a) Two-dimensional spectrum of the time-frequency analysis

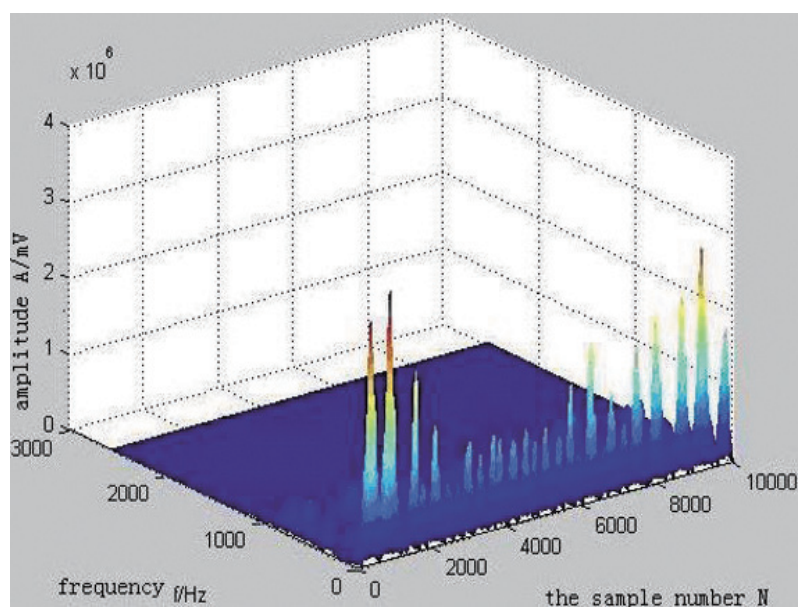

(b) Three-dimensional spectrum of the time-frequency analysis

Fig. 6. The time frequency analysis of a tooth root crack signal at $300 \mathrm{rpm}$.

From the comparison result of the Table 1, the SNR of the signal processed by wavelet threshold de-noising is larger than the SNR of the original signal, and the SNR of the signal processed by wavelet threshold de-noising (based on EMD) is much larger than the one simply used by the wavelet threshold de-noising. The fault feature is also more obvious, which is convenient to identify faults and multi-faults. From the subsequent time-frequency analysis result, it can be known that the wavelet threshold de-noising based on EMD has a larger advantage than the one simply used the wavelet threshold de-noising in time-frequency analysis. 


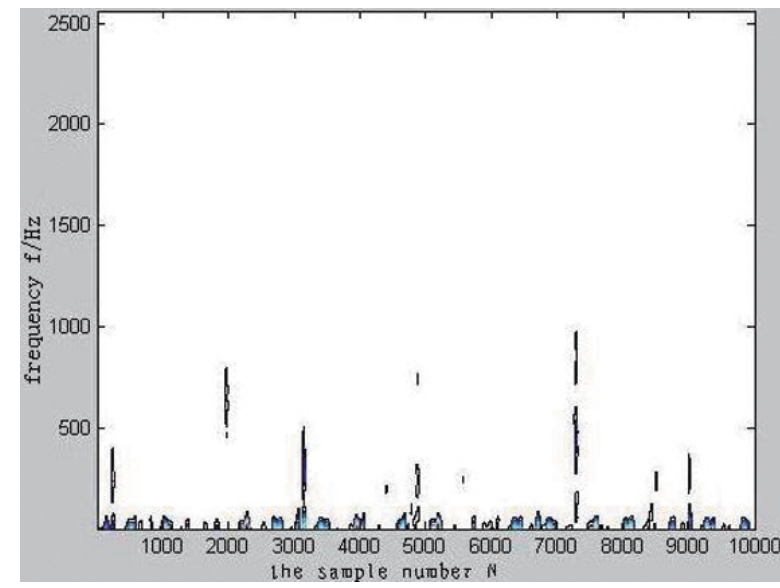

(a) Two-dimensional spectrum of the time-frequency analysis

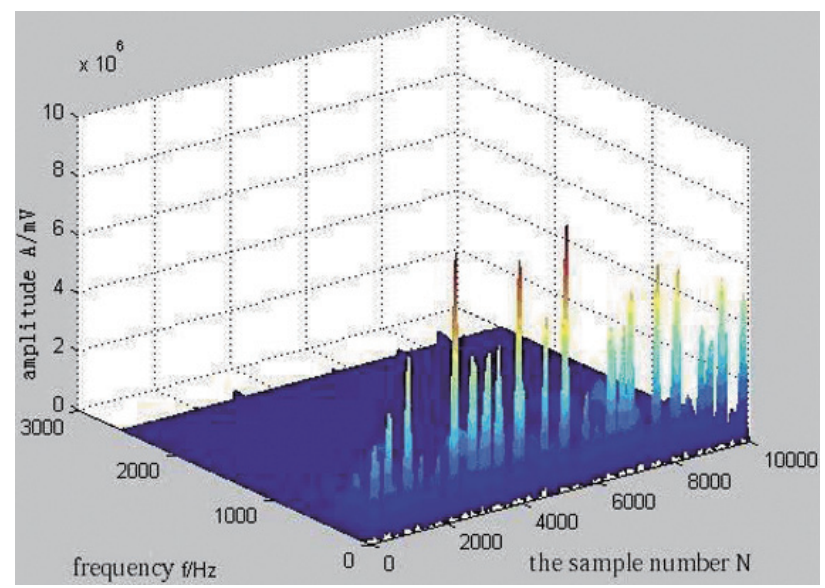

(b) Three-dimensional spectrum of the time-frequency analysis

Fig. 7. The time frequency analysis of a pitch circle crack signal at $300 \mathrm{rpm}$.

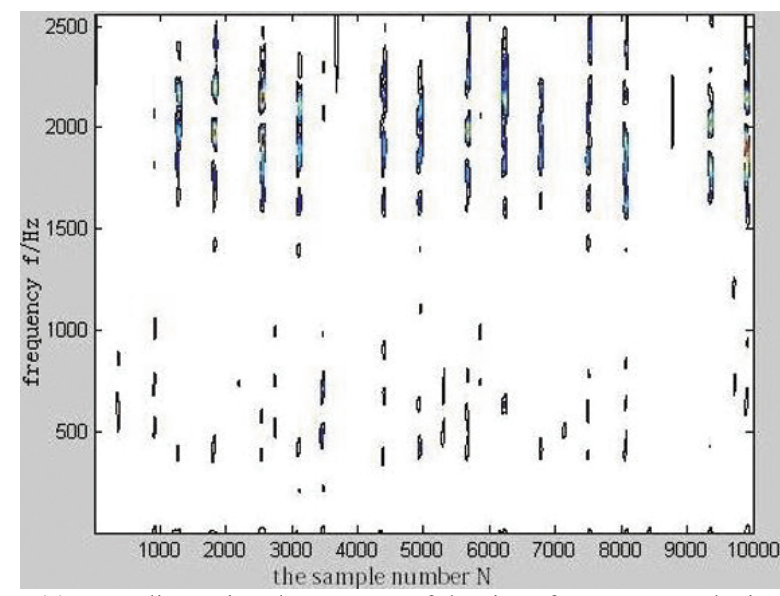

(a) Two-dimensional spectrum of the time-frequency analysis

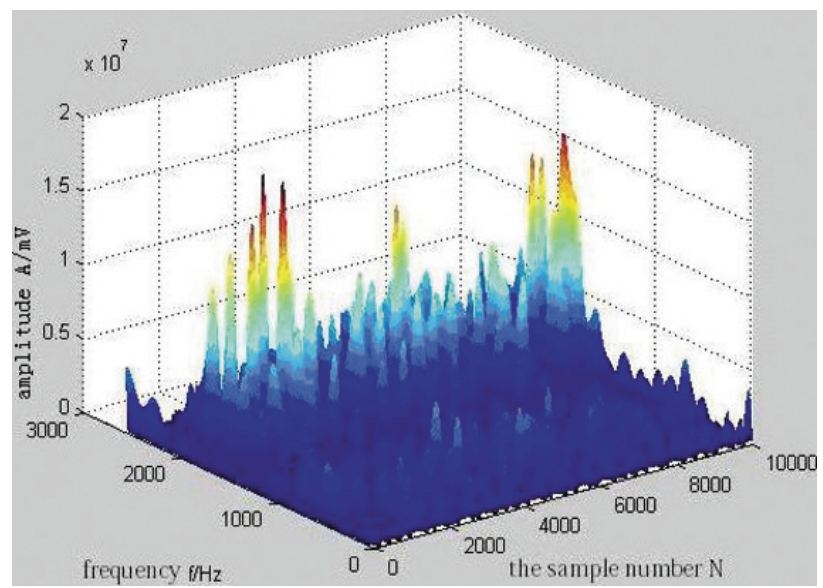

(b) Three-dimensional spectrum of the time-frequency analysis

Fig. 8. The time frequency analysis of a tooth wear signal at $300 \mathrm{rpm}$.

\subsection{Multi-fault diagnosis of a gear transmission system}

By firstly using a wavelet threshold de-noising based on EMD to pre-process the testing signal, and then analyzing the pre-processed signal by the time-frequency transformation, the different fault spectrums in different running conditions will be obtained, which can be used to identify and diagnose the faults. At $300 \mathrm{rpm}$, the rotation frequency is $5 \mathrm{~Hz}$ and the gear meshing frequency is $225 \mathrm{~Hz}$, The signal analysis result of the gear in no-fault is shown in Fig. 5 ; A low-frequency (rotation frequency), which can be seen from the two-dimensional figure and three-dimensional figures, is among $0-10 \mathrm{HZ}$, and there is also a great energy frequency (meshing frequency) at the vicinity of $230 \mathrm{HZ}$. At the same time, the analysis result of the tooth root crack signal is shown in Fig. 6: the vibration energy mainly concentrates on the rotation frequency because the crack only occurs in a tooth and the crack fault reflects in the rotation frequency. The analysis result of the pitch circle crack signal is shown in Fig. 7, for the same reason, the low-frequency energy has been strengthened, but because the crack is in different position, the low-frequency signal energy of the pitch circle crack is larger than the signal energy of the tooth root crack. The analysis result of a tooth wear signal is shown in Fig. 8. From the drawing, the energy bestrews the whole time-frequency domain. But the energy of high-frequency is reinforced, which is consistent with the low-frequency energy that is reinforced by tooth root crack, and the tooth wear strengthens the high-frequency energy. 


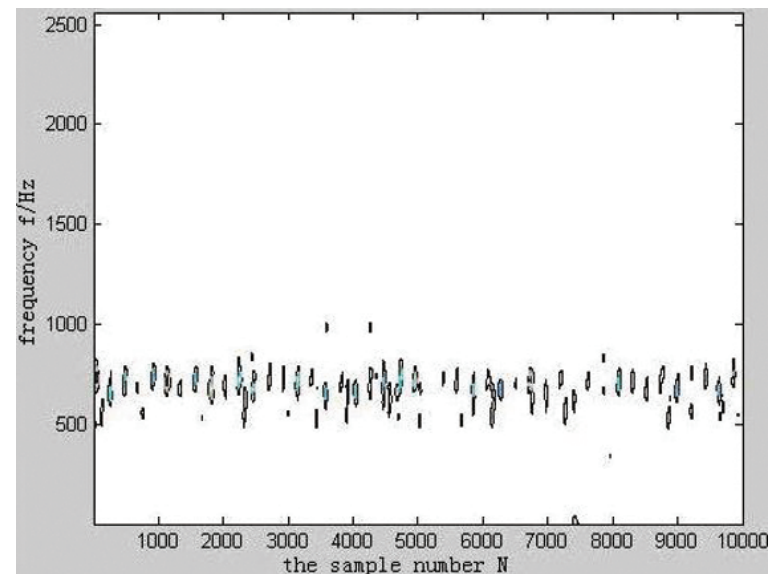

(a) Two-dimensional spectrum of the time-frequency analysis

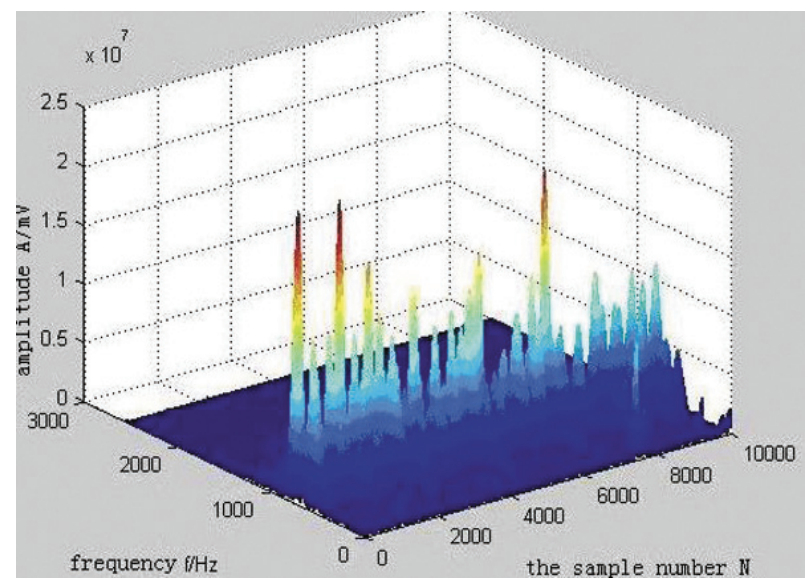

(b) Three-dimensional spectrum of the time-frequency analysis

Fig. 9. The time frequency analysis of a no fault signal at $900 \mathrm{rpm}$.

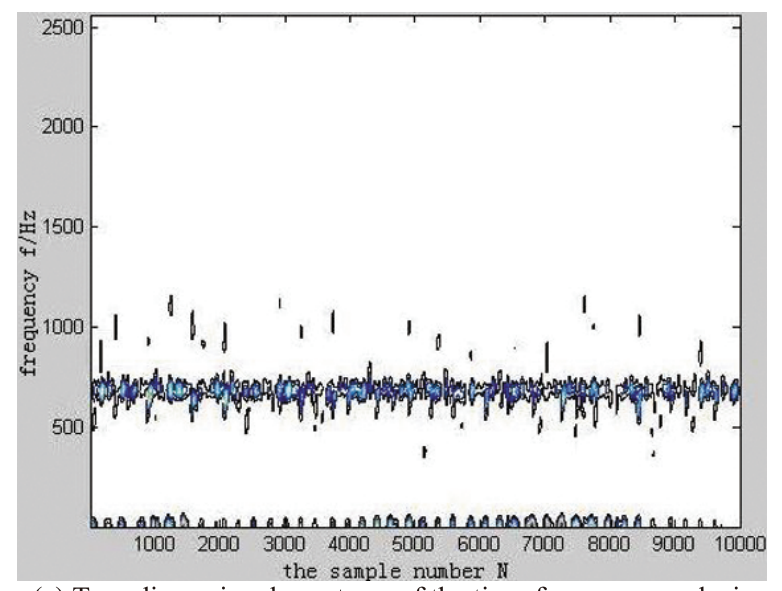

(a) Two-dimensional spectrum of the time-frequency analysis

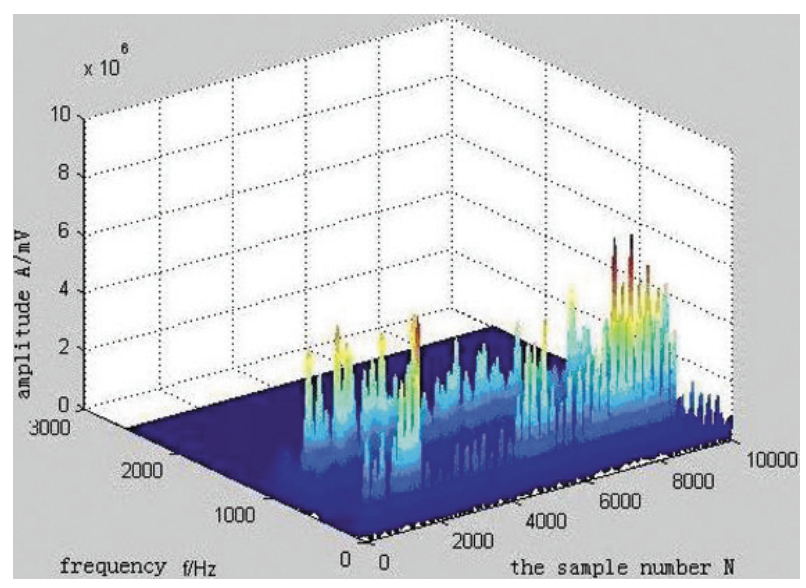

(b) Three-dimensional spectrum of the time-frequency analysis

Fig. 10. The time frequency analysis of a tooth root crack signal at $900 \mathrm{rpm}$.

At $900 \mathrm{rpm}$, the rotation frequency is $15 \mathrm{~Hz}$ and the gear meshing frequency is $675 \mathrm{~Hz}$, The signal analysis result of the gear without fault is shown in Fig. 9; the large amount of energy is mainly caused by the gear meshingfrequency, and only exists in the vicinity of $670 \mathrm{HZ}$. This result can be seen from the two- and three-dimensional figures. At high speed, the effect of gear meshing will reduce and the meshing impact between the teeth will increase; the feature drawing reflects that the energy of meshing frequency is very strong (Figs 10-12 show examples of this), but the energy of rotation frequency is submerged. The analysis result of the tooth root crack signal is shown in Fig. 10, and the analysis result of the pitch circle crack signal is shown in Fig. 11. Made clear from these figures, the low-frequency energy has been strengthened because the fault of single tooth crack reflects in the rotation frequency. Also, there can be seen that the low-frequency signal energy of the pitch circle crack is larger than the signal energy of the tooth root crack, and the distribution of tooth-root-crack signal is single (small range), while the pitch-circlecrack signal is scatteredly distributed (large distribution range). Both have their own features. The analysis result of the tooth-wear signal is shown in Fig. 12; the time-frequency domain is covered with the energy, the signal energy of both low-frequency and high-frequency is reinforced, which is consistent with the reinforced low-frequency energy that is strengthened by tooth root crack, and the tooth wear strengthens the high-frequency energy and the lowfrequency energy. It is noteworthy that, compared with tooth wear at $300 \mathrm{rpm}$; the high-frequency energy has a small increase while the low-frequency energy has an obvious increase. This shows that at the state of high speed, with the aggravation of tooth wear, the wear fault of some weak gear has a trend of developing into other fault forms. 


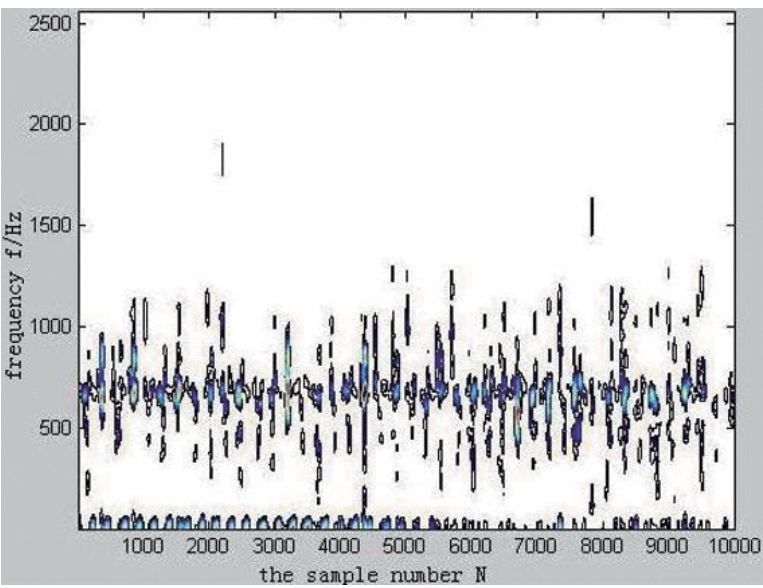

(a) Two-dimensional spectrum of the time-frequency analysis

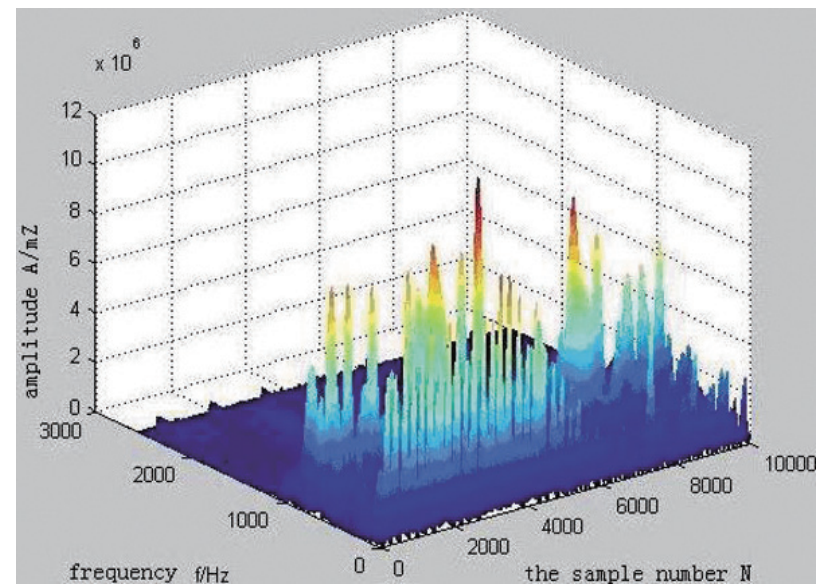

(b) Three-dimensional spectrum of the time-frequency analysis

Fig. 11. The time frequency analysis of a pitch circle crack signal at $900 \mathrm{rpm}$.

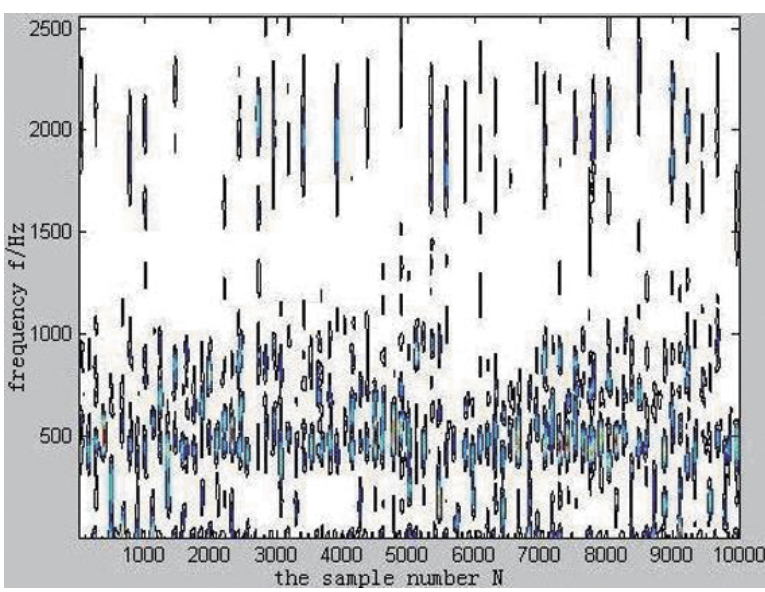

(a) Two-dimensional spectrum of the time-frequency analysis

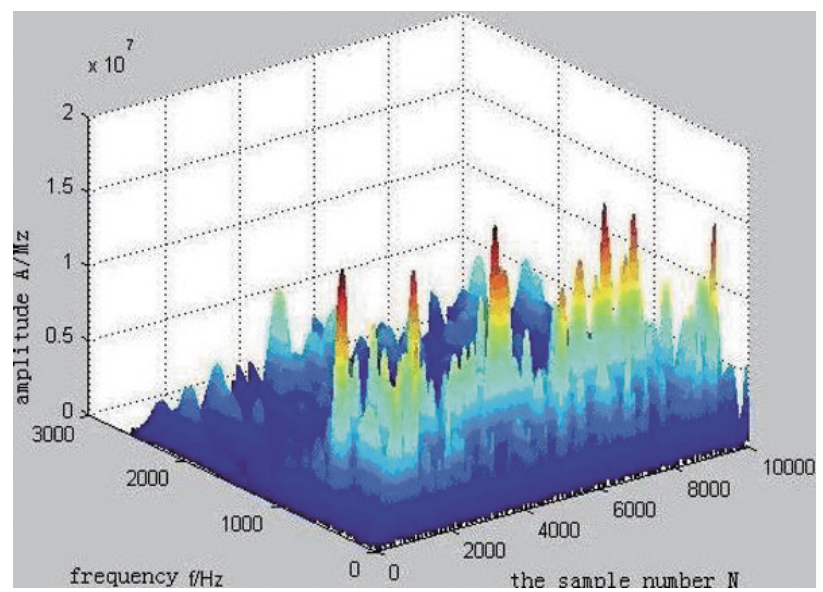

(b) Three-dimensional spectrum of the time-frequency analysis

Fig. 12. The time frequency analysis of a tooth wear signal at $900 \mathrm{rpm}$.

In a word, at the same speed, due to the differences of the frequency and the amplitude of the energy distribution which have their own features, three kinds of faults can be distinguished effectively. At different speeds, with the speed increasing, the frequency and the amplitude of the energy distributed in the time-frequency figure will change and become large. However, according to the feature of their energy distribution, every fault can still be identified. Therefore this method is better for fault detection and diagnosis.

\section{Development and realization of detection and diagnosis system}

\subsection{Mixed programming of MATLAB and $V C++$ using COM module technology}

MATLAB is a set of numerical calculation software developed by Math Works Corporation of the USA. It can achieve computing and graphics processing of many areas, such as numerical analysis, optimization, statistics, finding numerical solution of partial differential equations, automatic control, signal processing, and image processing. MATLAB taken matrix as the basic data structure is very suitable for large-scale numerical calculations. However, MATLAB is an interpreted language, so its execution speed is relatively slow. $\mathrm{C}++$ is a compiled language. After 


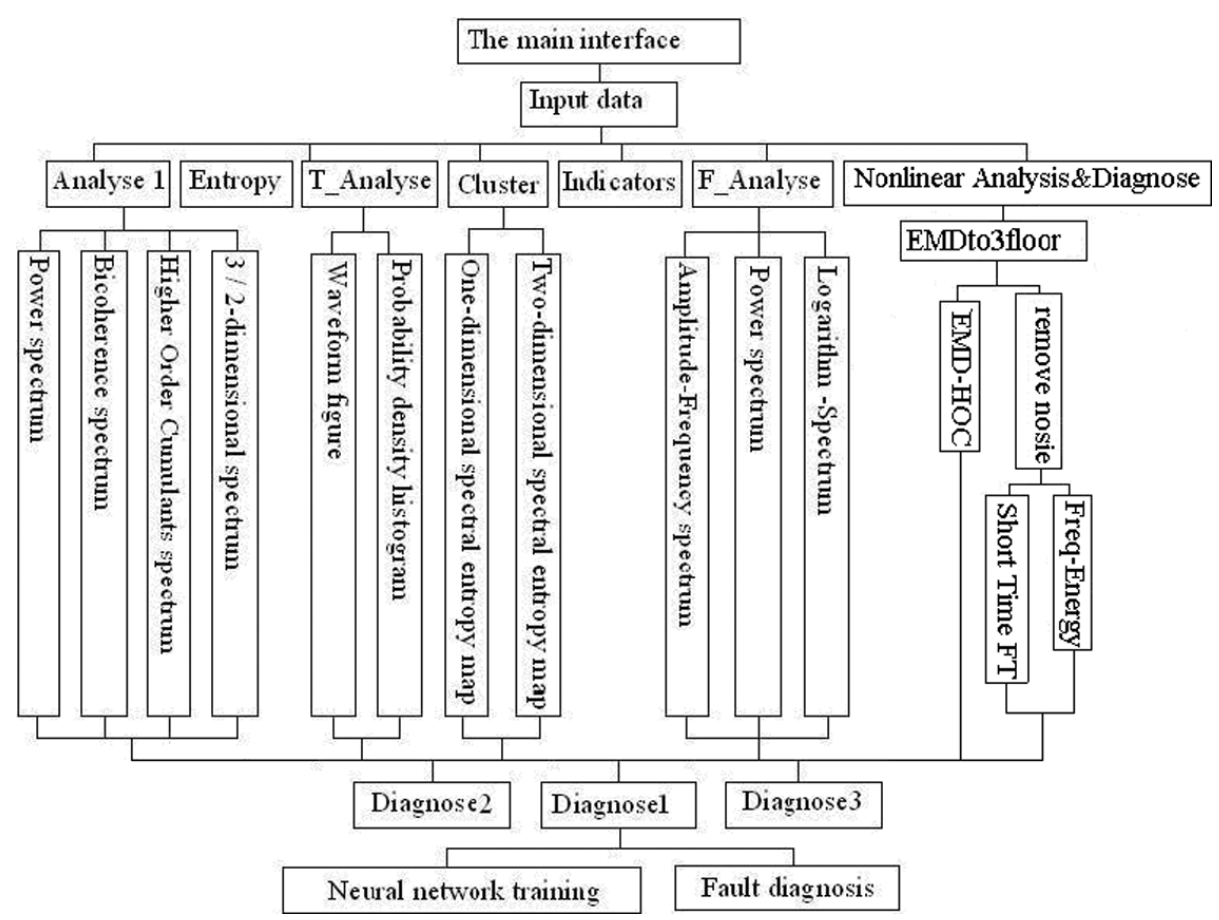

Fig. 13. Structure of a software system.

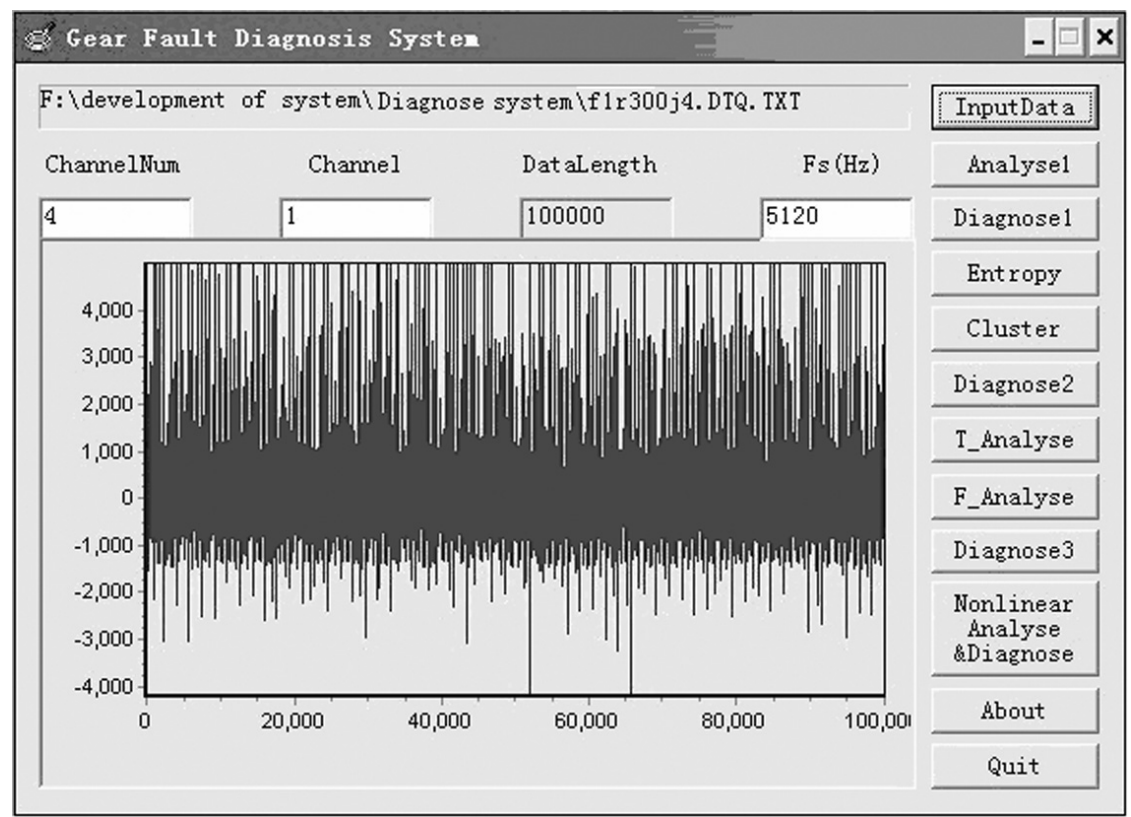

Fig. 14. The main interface of software and the display of interface after importing data.

being compiled, the program taken as binary code will be executed, so its execution speed is quite high, and this advantage will become more obvious when performing the multi-level nested loops operation. Visual $\mathrm{C}++$ is a visual development tool, which as well as a windows operating system comes from Microsoft Corporation, so it has a unique advantage among application software development platforms. However, Visual $\mathrm{C}++$ is not good at 


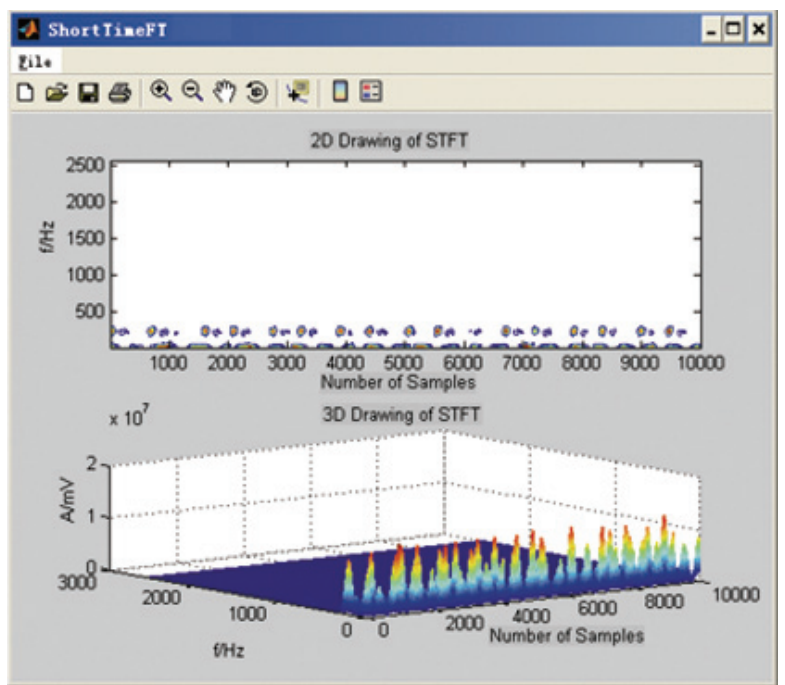

(a) The short-time Fourier transform of a no fault signal at $300 \mathrm{rpm}$

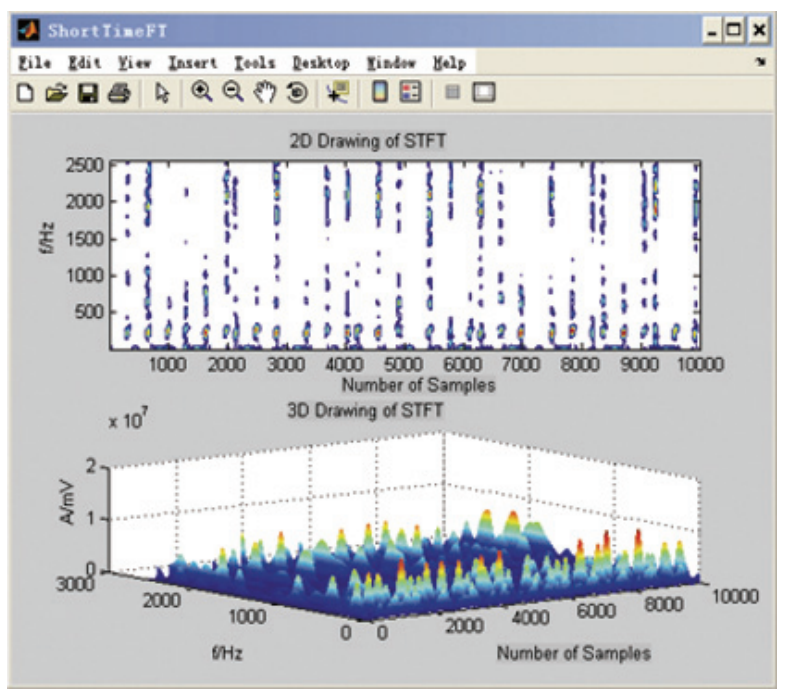

(c) The short-time Fourier transform of a tooth wear signal at $300 \mathrm{rpm}$

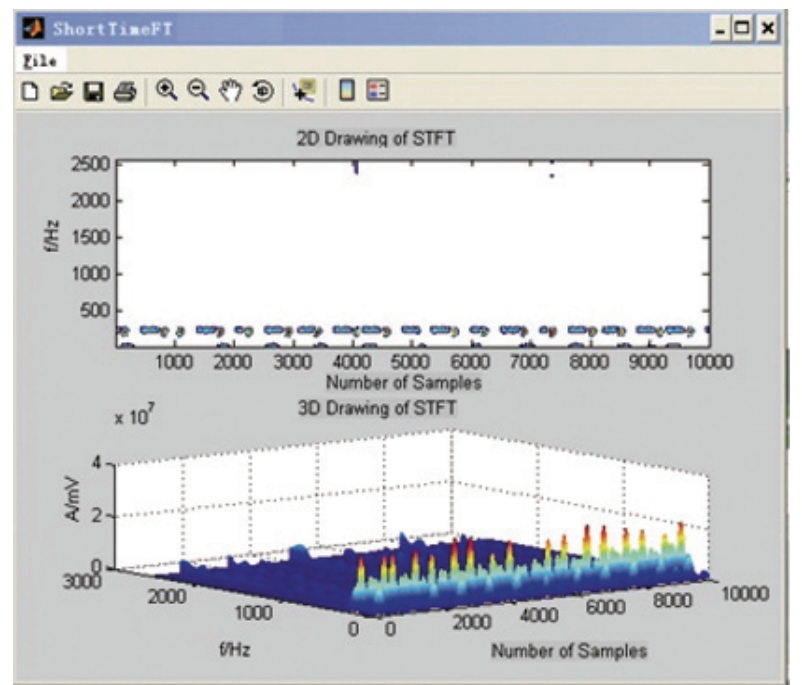

(b) The short-time Fourier transform of a tooth root short crack signal at $300 \mathrm{rpm}$

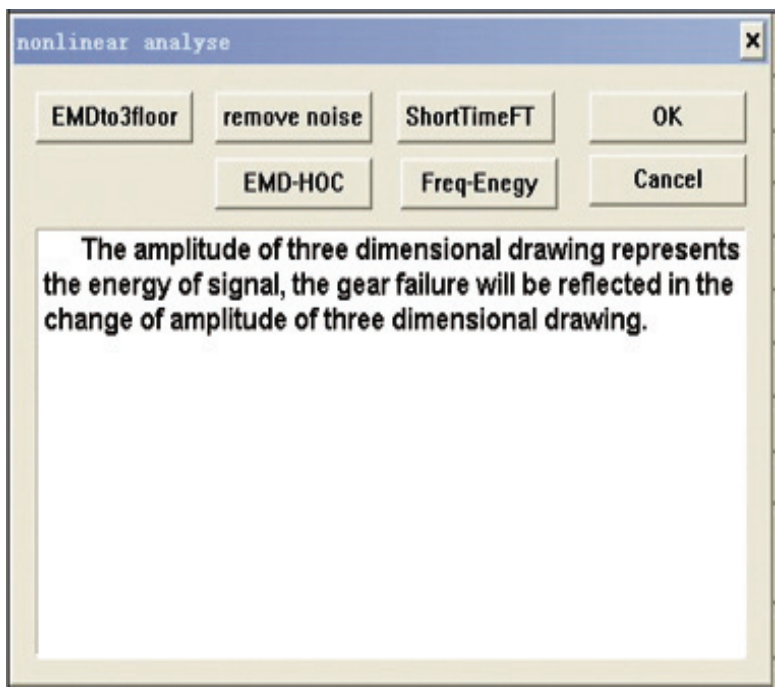

(d) The main interface and the note of nonlinear analysis

Fig. 15. The analysis interface of short-time Fourier transform.

achieving complex numerical calculation like MATLAB. Therefore, this paper effectively combines the advantages of MATLAB and Visual $\mathrm{C}++$.

The key thought is that an application system is cut into many modules with a certain independence of function. When the exploitation of all components is completed, put them together to get the complete application system. Through the interfaces, the components interoperate with each other to co-work. When alterations are required, we need not change the whole system but change the affected components to reassemble the new upgrade software.

COM (Component Object Module) it is a common object interface. According to the interface standard, any language can call it. Com Builder released in MATLAB 6.5 can change a program written in MATLAB to COM components for other languages call. The method is simple and versatile; almost any function of MATLAB can be used. According to the characteristics of the system developed in this paper, the COM module technology is chosen for system exploitation, which changes the procedures of each signal analysis and processing algorithm written in MATLAB into COM components. After completion of system exploitation, when a better signal analysis and processing algorithms appear, it only need update or add the relevant modules. 


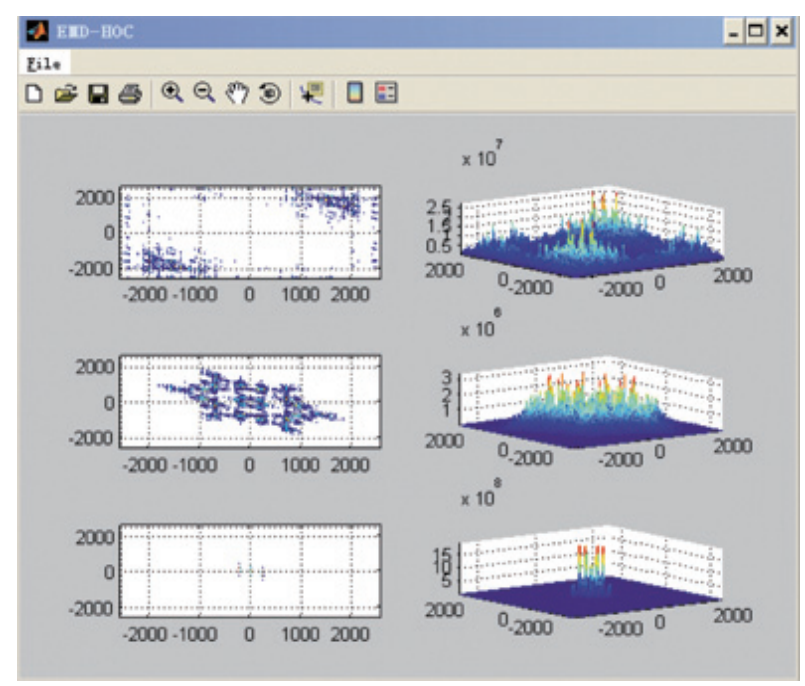

(a) Third-order cumulant spectrum based on EMD of no fault at $300 \mathrm{rpm}$

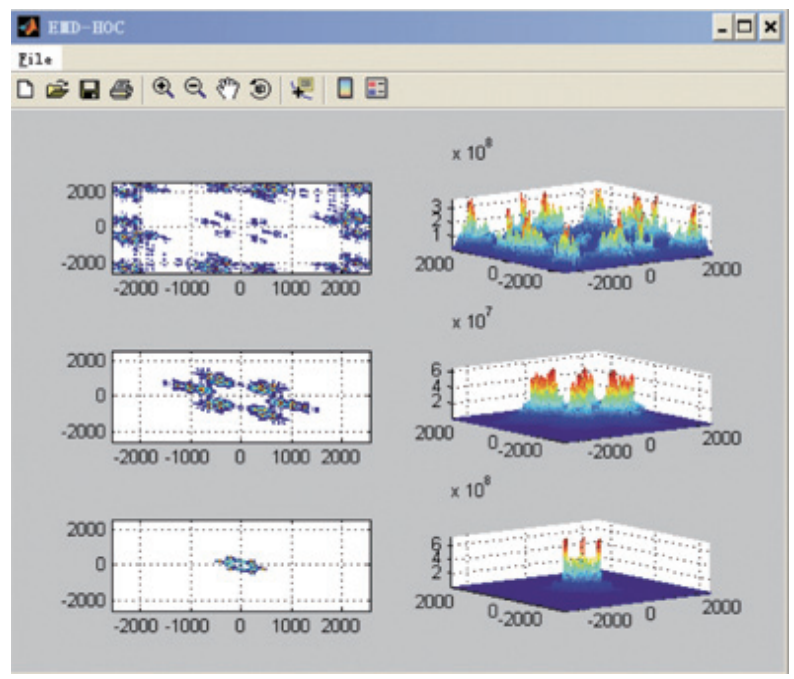

(c) Third order cumulant spectrum based on EMD of tooth wear at 300 rpm

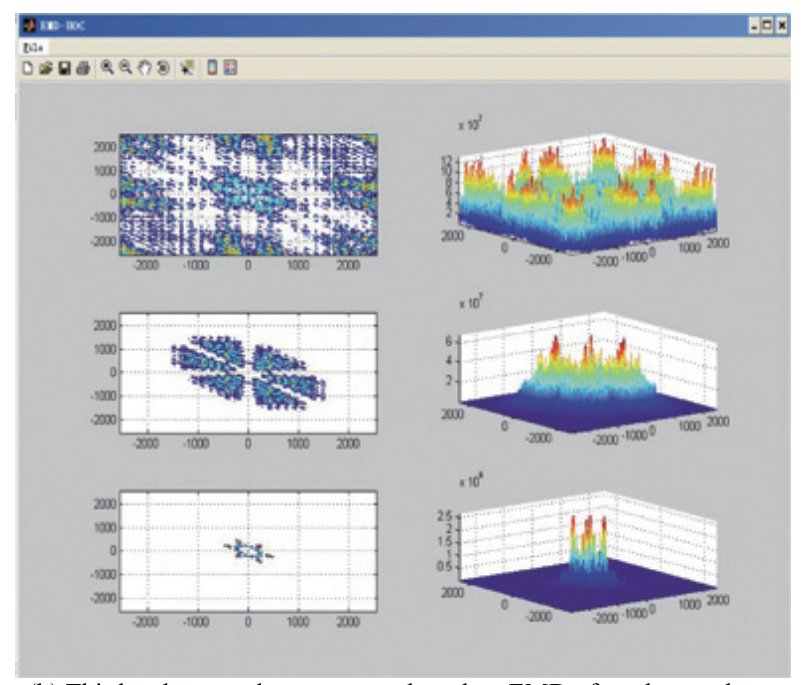

(b) Third-order cumulant spectrum based on EMD of tooth root short crack at $300 \mathrm{rpm}$

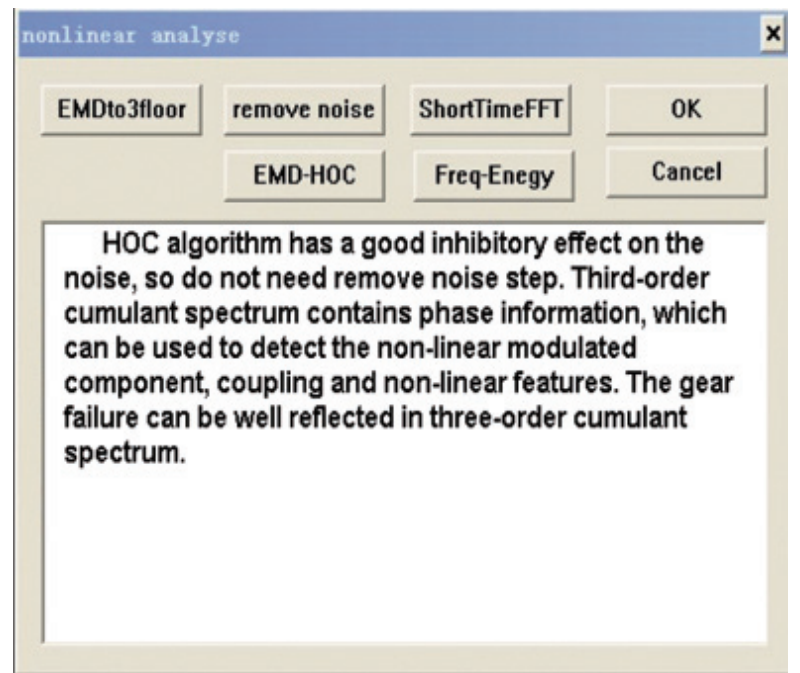

(d) The main interface and the note of EMD-HOC

Fig. 16. The analysis interface of EMD-HOC.

\subsection{Construction of system}

The software, which could be run without MATLAB software environment, can be developed by COM module technology. $\mathrm{VC}++$ is responsible to open the document and for the main interface, and MATLAB is responsible for numerical computation, signal processing, the graphics display and other functions. The *.m file can be converted into DLL that VC++ can call by MATLAB Builder for COM, and finally the development of the system is completed. The system, which has seven functional interfaces, chiefly includes: the main function interface; the various interfaces of gear vibration signal analysis; the various interfaces of gear vibration signal diagnosis and identification; and the interfaces of gear vibration signal detection and monitoring. Each interface includes a corresponding sub-interface with analysis and diagnosis function, as shown in Fig. 13. The main interface is shown in Fig. 14. It mainly introduces the signal acquired from the actual gear transmission testing system in order to facilitate subsequent analysis, identification, and diagnosis. The top column is used to show the physical address of imported data, "ChannelNum" is used to set the total channel number of data files, "Channel" is used to set the channel sequence of 

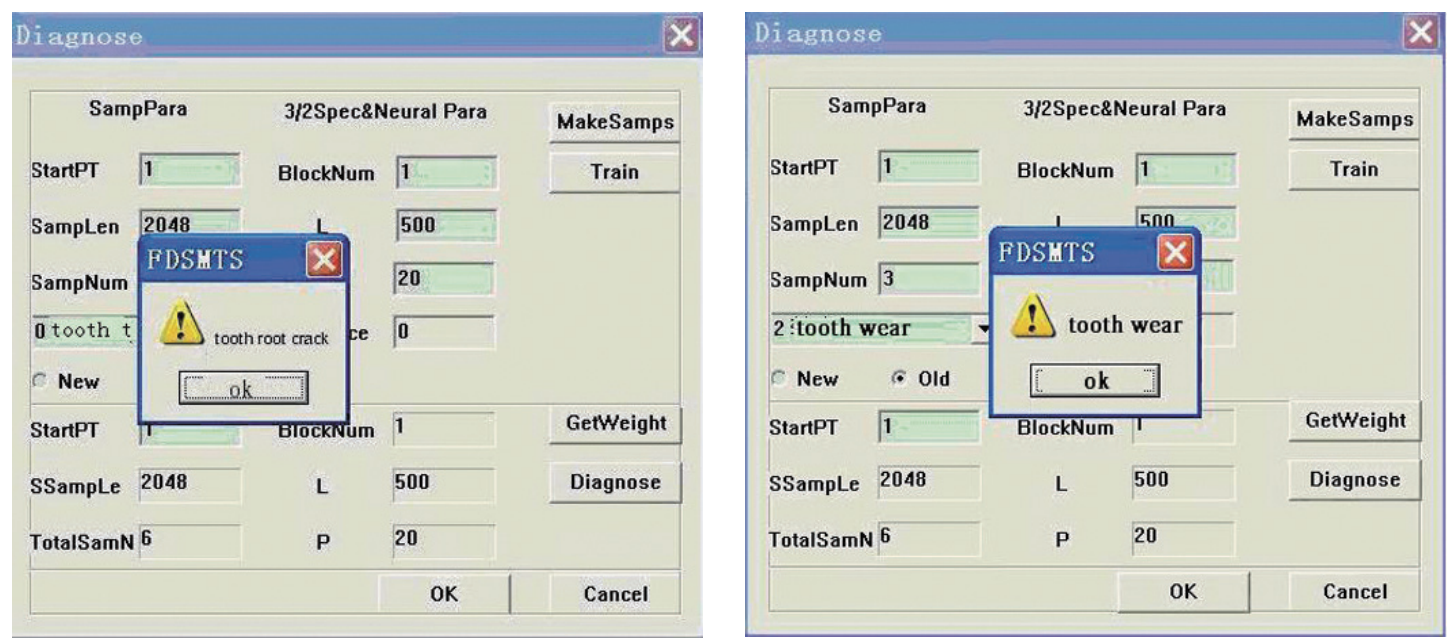

Fig. 17. Detection and diagnosis result display interface of the VI system.

imported data, "Data Length" is used to display imported data length, and "Fs (Hz)" is used to set the sampling frequency. If the data format is correct, the graph-displayed frame will display the waveform of the imported data. The twelve buttons on the right are used for signal processing, calculation and analysis, feature extraction, identification, diagnosis, etc.

\subsection{Diagnosis system operation and diagnosis result output}

The method of wavelet threshold de-noising which combined with EMD is used to pre-process the signal. It then gives this signal a process of time-frequency analysis, so the time frequency analysis spectrum of different faults will be obtained in different running conditions, which can be used to identify and diagnose these faults. Instructions on how to input a parameters in the main interface of the system is shown in Fig. 14. Click the "Input Data" button to open the dialog box of data file and select the date to be analyzed, then set the parameters. If the data format is correct, the whole waveform of the imported data will be displayed in the graphical display box, which indicates that the data is successfully imported. Figure 14 displays the main interface of the system after imported the fourchannels test data. The button of "Nonlinear Analyse and Diagnose" is used to analyze and diagnose the nonlinear system data. Clicking this button will pop up a new window "nonlinear analyse", as shown in Fig. 15(d). There are five function buttons, "EMDto3floor", "remove noise", "EMD-HOC", "Short Time FT", and "Freq-Energy". At first, only the button "EMDto3floor" is active; this is because the function of this button is the necessary step to achieve the function of other buttons. Clicking it will decompose the original signal into three levels by EMD, and then the "remove noise" button will be actived. Clicking it will achieve the function of de-noising the original signal. At the same time, the button of "ShortTimeFT" is activated; clicking it will give the de-noised signal a short-time Fourier transform.

As show in Figs 15(a)-(c), clicking the button of "ShortTimeFT" will pop-up a window of ShortTimeFT. This window displays the short-time Fourier transform of the de-noised signal. Meanwhile, as shown in Fig. 15(d), the note in the text of the nonlinear analysis window also makes a corresponding change.

The window of EMD-HOC will pop-up when the EMD-HOC button is clicked on, as shown in Figs 16(a)-(c). The figure of the two-dimensional and three-dimensional drawings of the high-order cumulant spectrum, obtained by decomposing the original signal used EMD, has been shown in the window. Meanwhile, the note in the text of the nonlinear analysis window also makes a corresponding change, as shown in Fig. 16(d).

The interface of the diagnosis result has been shown in Fig. 17 (tooth wear and tooth root crack), which shows the diagnosis result of the fault, such as no fault, tooth root crack, pitch circle crack, tooth surface pitting and tooth wear. The actual testing result shows that the system works well. 


\section{Conclusions}

(1) Through the way of effectively combining the EMD with the wavelet threshold de-noising, we exert their advantages and propose a characteristic processing method of wavelet threshold de-noising based on EMD. By this method, the wavelet threshold de-noising does not act on the whole signal but only the high-frequency component of the signal. The method largely overcomes the defect of simply using the method of wavelet threshold de-noising and improves the accuracy of the analysis, which will highlight the fault feature of the signal. Based on the de-noising processing, the method combined with the time-frequency analysis can effectively identify different types of gear faults, such as tooth root crack, pitch circle crack and tooth wear, which can not only applied to a gear transmission system but can also be extended to other fault recognition and diagnosis fields.

(2) By the diagnosis algorithm combined with the advantages of MATLAB and $\mathrm{VC}++$, the online damage detection and diagnosis software system of gear system was successfully developed, which possesses some functions, including the introduction of gear vibration signals, signal processing, feature extraction, graphics displays, fault detection, and diagnosis. Based on validation of testing results, the system can be effectively used in gear fault detection and diagnosis.

\section{Acknowledgments}

This research is sponsored by the Natural Science Fund of China (NSFC) (Grant No.51275422), the Aviation Science Fund of China (01I53073), the Shaanxi Province Natural Science Foundation (2004E219) and the Graduate Carve Out Seed Fund of Northwestern Polytechnical University (Z2013032).

\section{References}

[1] Y. Li, J. Zhang, L. Dai et al., Auditory-model based feature extraction method for mechanical faults diagnosis, Chinese Journal of Mechanical Engineering 23(3) (2010), 391-397.

[2] R. Shao, H. Liu and Y. Xu, Fault detection and diagnosis of gear system based on higher order cumulants, Chinese Journal of Mechanical Engineering 44(6) (2008), 161-168.

[3] R. Shao, Y. Shen and J. Sun, Dynamic analysis of variable natural characteristic for gear decelerator, Acta Aeronautica Et Astronautica Sinica 22(1) (2001), 64-68.

[4] P. Ju, S. Qin and Y. Qin, Research on earlier fault diagnosis of gear by method of multi-resolution empirical mode decomposition and frequency domain averaging, Journal of Vibration and Shock 28(5) (2009), 97-101.

[5] J. Sandberg and M. Hansson-Sandsten, Optimal stochastic discrete time-frequency analysis in the ambiguity and time-lag domain, Signal Processing 90(7) (2010), 2203-2211.

[6] J.J. Zhang, A. Papandreou-Suppappola and B. Gottin, Time-frequency characterization and receiver waveform design for shallow water environments, IEEE Transactions on Signal Processing 57(8) (2009), 2973-2985.

[7] A.C. To, J.R. Moore and S.D. Glaser, Wavelet denoising techniques with applications to experimental geophysical data, Signal Processing 89(2) (2009), 144-160.

[8] X. Chen and B. Li, Acoustic emission method for tool condition monitoring based on wavelet analysis, International Journal of Advanced Manufacturing Technology 33(9-10) (2007), 968-976.

[9] C. He, S. Liu, Z. Liu et al., The application of the wavelet de-noising in detect the steel strand defects, Chinese Journal of Mechanical Engineering 44(7) (2008), 118-122.

[10] T. Yang, Y. Gao and X. Kong, Investigation on wavelet-based method of fault diagnosis for a piston pump, Chinese Journal of Mechanical Engineering 41(2) (2005), 112-116.

[11] K. Guo, X. Zhang, H. Li and G. Meng, Application of EMD method to friction signal processing, Mechanical Systems and Signal Processing 22(1) (2008), 248-259.

[12] J. Cheng, D. Yu and Y. Yang, A fault diagnosis approach for roller bearings based on EMD method and AR model, Mechanical Systems and Signal Processing 20(2) (2006), 350-362.

[13] F. Wu and L. Qu, Diagnosis of subharmonic faults of large rotating machinery based on EMD, Mechanical Systems and Signal Processing 23(2) (2009), 467-475.

[14] Z. Li and H. Xu The study of wavelet threshold denoising based on EMD, The Computer Simulation 26(9) (2009), 325-328.

[15] H. Li, X. Deng and H. Dai, Structural damage detection using the combination method of EMD and wavelet analysis, Mechanical Systems and Signal Processing 21(1) (2007), 298-306. 
[16] C. Wang, Z. Zhou, P.J. Unsworth and P. Igic, Current space vector amplitude fluctuation based sensor less speed measurement of induction machines using short time Fourier transform, industrial electronics (IECON), 34th Annual Conference of IEEE, Orlando FL, USA (1-5 Jan 2008), 1869-1874.

[17] D. Zhou, Short-time Fourier transform and the ascension wavelet transform applied in analyze the pulse signal, MA Dissertation, Chongqing University, 2008.

[18] K.P. Kuldip and D.A. Leigh, On the usefulness of STFT phase spectrum in human listening tests, Speech Communication 45(2) (2005), $153-170$.

[19] S.M. Debbal and F. Bereksi-Reguig, Time-frequency analysis of the first and the second heartbeat sounds, Applied Mathematics and Computation 184(2) (2007), 1041-1052.

[20] R. Shao, H. Liu and J. Hu, Fault diagnostic VI system based on higher order cumulants, Chinese Journal of Scientific Instrument 29(1) (2008), 5-10.

[21] G. Xia, J. Feng and R. Ren, Study on the measurement of aircraft inverter output characteristics based on virtual instrument, Chinese Journal of Scientific Instrument 31(11) (2010), 2556-2570.

[22] H. Zhou and C. Gen, Design and application of virtual instrument system software architecture description language, Chinese Journal of Mechanical Engineering 23(2) (2001), 7-11.

[23] J. Baili, S. Lahouar and M. Hergli, GPR signal de-noising by discrete wavelet transform, NDT \& E International 42(8) (2009), 696-703.

[24] G. Rilling, P. Flandrin and P. Gonçalves, On empirical mode decomposition and its algorithms, IEEE-EURASIP Workshop on Nonlinear Signal and Image Processing, NSIP-03, Grade I (2003), 8-11.

[25] Y. Kopsinis and S. McLaughlin, Development of EMD based denoising methods inspired by wavelet thresholding, IEEE Transactions on Signal Processing: A publication of the IEEE Signal Processing Society 57(4) (2009), 1351-1362.

[26] J. Zhong and Y. Huang, Time-frequency representation based on an adaptive short-time Fourier transform, IEEE Transactions on Signal Processing 58(10) (2010), 5118-5128. 

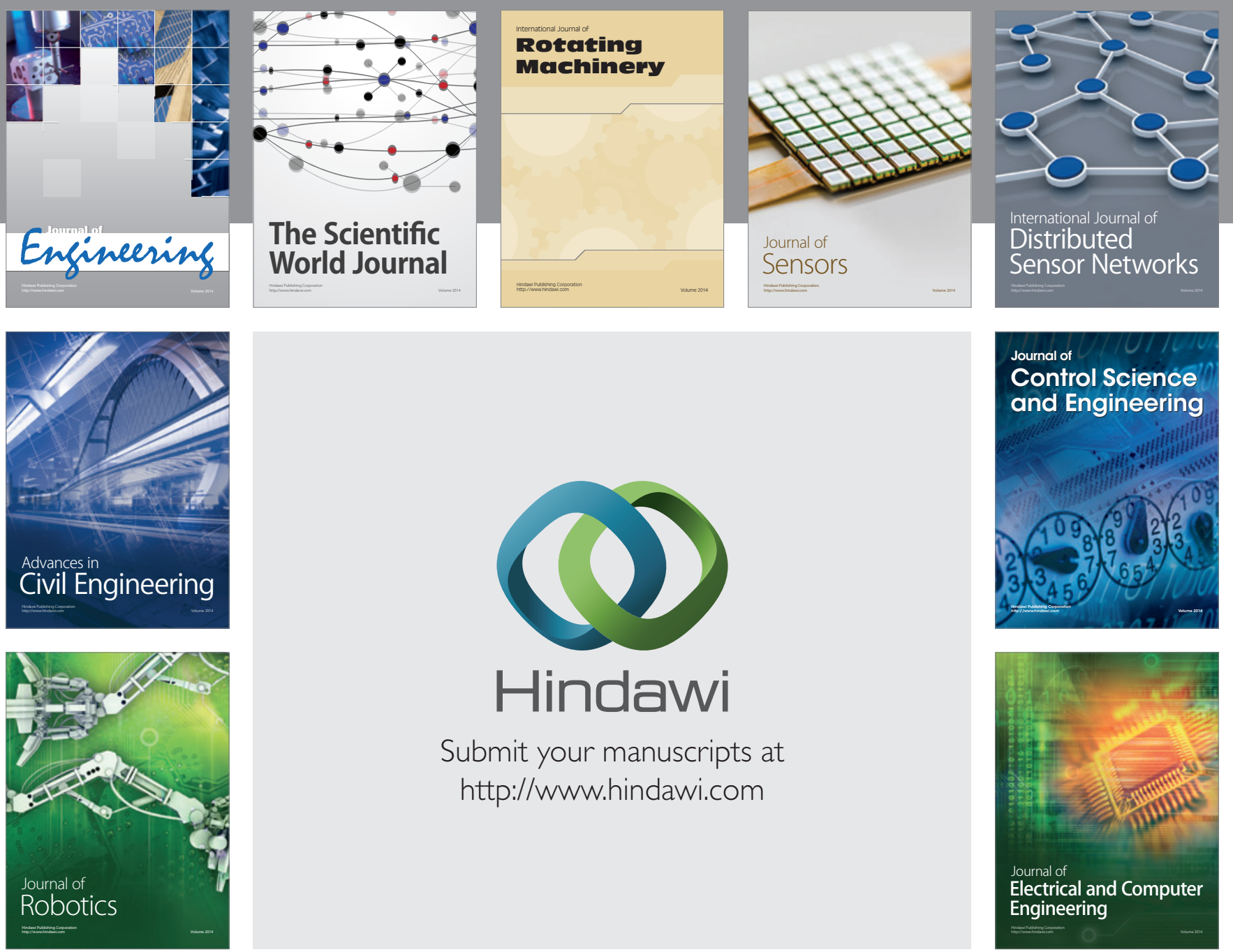

Submit your manuscripts at

http://www.hindawi.com
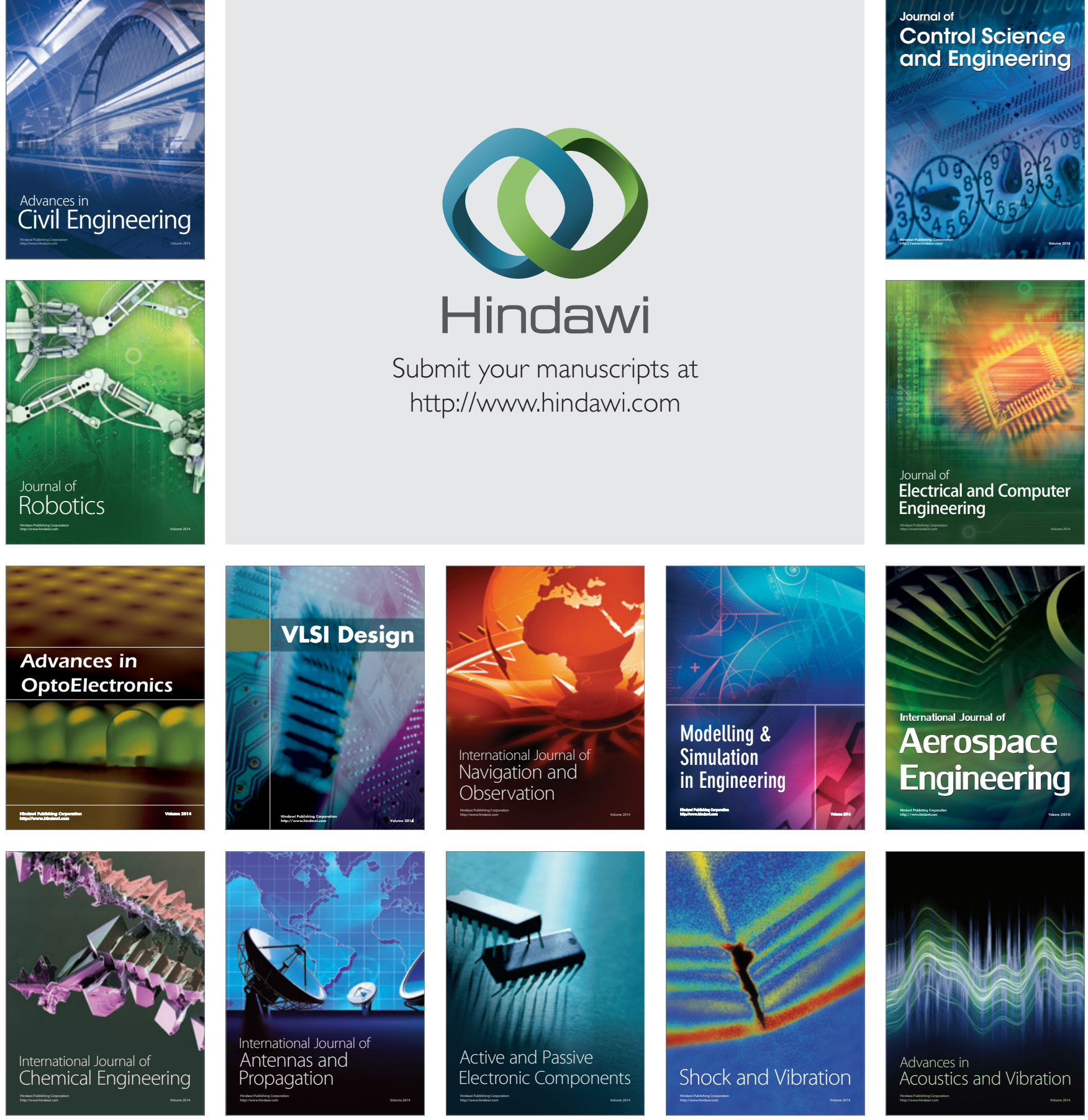